\title{
Ranking Quantitative Resistance to Septoria tritici Blotch in Elite Wheat Cultivars Using Automated Image Analysis
}

\author{
Petteri Karisto ${ }^{\dagger}$ Andreas Hund, Kang Yu, Jonas Anderegg, Achim Walter, Fabio Mascher, \\ Bruce A. McDonald, and Alexey Mikaberidze
}

First, seventh, and eighth authors: Plant Pathology Group, Institute of Integrative Biology, ETH Zurich, Zurich, Switzerland; second, third, fourth, and fifth authors: Crop Science Group, Institute of Agricultural Sciences, ETH Zurich, Zurich, Switzerland; and sixth author: Crop Breeding and Genetic Resources, Department of Plant Breeding, Agroscope, Nyon, Switzerland.

Accepted for publication 3 December 2017.

\begin{abstract}
Quantitative resistance is likely to be more durable than major gene resistance for controlling Septoria tritici blotch (STB) on wheat. Earlier studies hypothesized that resistance affecting the degree of host damage, as measured by the percentage of leaf area covered by STB lesions, is distinct from resistance that affects pathogen reproduction, as measured by the density of pycnidia produced within lesions. We tested this hypothesis using a collection of 335 elite European winter wheat cultivars that was naturally infected by a diverse population of Zymoseptoria tritici in a replicated field experiment. We used automated image analysis of 21,420 scanned wheat leaves to obtain quantitative measures of conditional STB intensity that were precise, objective, and reproducible. These measures allowed us to explicitly separate resistance affecting host damage from resistance affecting pathogen reproduction, enabling us to confirm that these resistance traits are largely independent. The cultivar rankings based on host damage were different from the rankings based on pathogen reproduction, indicating that the two forms of

resistance should be considered separately in breeding programs aiming to increase STB resistance. We hypothesize that these different forms of resistance are under separate genetic control, enabling them to be recombined to form new cultivars that are highly resistant to STB. We found a significant correlation between rankings based on automated image analysis and rankings based on traditional visual scoring, suggesting that image analysis can complement conventional measurements of STB resistance, based largely on host damage, while enabling a much more precise measure of pathogen reproduction. We showed that measures of pathogen reproduction early in the growing season were the best predictors of host damage late in the growing season, illustrating the importance of breeding for resistance that reduces pathogen reproduction in order to minimize yield losses caused by STB. These data can already be used by breeding programs to choose wheat cultivars that are broadly resistant to naturally diverse $Z$. tritici populations according to the different classes of resistance.
\end{abstract}

Zymoseptoria tritici (Roberge ex Desm.) Quaedvl. \& Crous (formerly Mycosphaerella graminicola (Fuckel) J. Schröt.) is a fungal pathogen that poses a major threat to wheat production, particularly in temperate areas (Dean et al. 2012; Jorgensen et al. 2014). It infects wheat leaves, causing the disease Septoria tritici blotch (STB). Yield losses caused by STB can be 5 to $10 \%$, even when resistant cultivars and fungicides are used in combination. Approximately $\$ 1.2$ billion is spent annually in Europe on fungicides targeted mainly toward STB control (Torriani et al. 2015). Z. tritici populations in Europe carry a high degree of fungicide resistance (reviewed by Fones and Gurr 2015; Torriani et al. 2015). In several cases, fungicides repeatedly lost their efficacy only a few years after their introduction due to rapid emergence of fungicide-resistant strains of Z. tritici (Fraaije et al. 2005; Griffin and Fisher 1985; Torriani et al. 2009). Resistance to azoles, an important class of fungicides that is widely used to control STB, has been growing steadily over the last 20 years in Europe (Cools and Fraaije 2013; Zhan et al. 2006) and appeared recently in North America (Estep et al. 2015). Therefore, STB-resistant wheat cultivars have become an important breeding objective to enable more effective management of the disease (McDonald and Mundt 2016). Major resistance genes

†Corresponding author: P. Karisto; E-mail: petteri.karisto@usys.ethz.ch

Funding: P. Karisto and A. Mikaberidze gratefully acknowledge financial support from the Swiss National Science Foundation through the Ambizione grant PZOOP3 161453 .

*The $\boldsymbol{e}$-Xtra logo stands for "electronic extra" and indicates that five supplementary files and 11 supplementary tables are published online.

(C) 2018 The American Phytopathological Society such as Stb6 (Brading et al. 2002) provide nearly complete resistance against a subset of $Z$. tritici strains carrying the wild-type AvrStb6 allele (Zhong et al. 2017) but, as found for fungicides, major resistance often breaks down a few years after it is introduced (Cowger et al. 2000). Quantitative resistance may be conferred by a large number of quantitative trait loci (QTL) with small and additive effects that can be combined to provide high levels of disease resistance (Kou and Wang 2010; McDonald and Linde 2002; Mundt 2014; Poland et al. 2009; St. Clair 2010). Quantitative resistance is thought to be more durable and, hence, deserves more attention from breeders (McDonald and Linde 2002; Mundt 2014; St. Clair 2010).

To enable breeding for quantitative resistance to STB, we need to comprehensively analyze the quantitative distribution of its associated phenotypes, which is much more difficult than phenotyping major gene resistance that typically shows a binomial distribution. This challenge was recognized more than 40 years ago and a number of studies were conducted to evaluate quantitative resistance to STB under field conditions using artificial inoculation (Brown et al. 2001; Eyal 1992; Miedaner et al. 2013; Rosielle 1972; Shaner and Finney 1982) and natural infection (Kollers et al. 2013b; Miedaner et al. 2013; Rosielle 1972; Shaner et al. 1975). Resistance to STB was also investigated on detached leaves with artificial inoculations (Chartrain et al. 2004). Several studies performed visual scoring of quantitative resistance only once during the growing season (Eyal 1992; Miedaner et al. 2013; Rosielle 1972; Shaner and Finney 1982), whereas other studies included two or more time points (Brown et al. 2001; Kollers et al. 2013b; Shaner et al. 1975). One of the most comprehensive early studies screened 7,500 wheat varieties, including 2,000 durum wheat cultivars, to select the 460 most resistant varieties for more detailed visual scoring (Rosielle 1972). 
Understanding the infection cycle of STB is important to distinguish and measure the most relevant aspects of quantitative resistance to the disease. $Z$. tritici spores germinate on wheat leaves and penetrate the leaves through stomata (Kema et al. 1996). After penetration, the fungus grows for several days within leaves without producing visible symptoms. During this latent phase, Z. tritici mycelium grows in the apoplast and invades the host mesophyll around the position of the initial penetration (Duncan and Howard 2000). After 10 to 14 days of asymptomatic growth, the fungus becomes necrotrophic, necrotic lesions appear in the invaded host tissue, and asexual fruiting bodies called pycnidia begin to form (Duncan and Howard 2000; Kema et al. 1996). In the dead host tissue, the fungus continues to grow saprotrophically and produces sexual fruiting bodies called pseudothecia 25 to 30 days after infection (Sánchez-Vallet et al. 2015). Whether Z. tritici is best referred to as a hemibiotroph or a latent necrotroph remains unclear (Sánchez-Vallet et al. 2015). Asexual pycnidiospores are usually spread by rain splash whereas sexual ascospores are spread by wind. The pathogen typically undergoes five to six rounds of asexual reproduction and one to two rounds of sexual reproduction per growing season.

Studies by Zhan et al. $(1998,2000)$ indicated that approximately $66 \%$ of infections on flag leaves came from asexual spores, while approximately $24 \%$ came from ascospores originating from within the infected field and approximately $10 \%$ of flag leaf infections were immigrants from surrounding fields. Thus, pathogen asexual reproduction is the most important factor explaining infection on flag leaves but a significant fraction of flag leaf infections can originate from airborne ascospores coming from within or outside of the field. The amount of necrosis induced by STB on the uppermost leaves determines yield losses (Brokenshire 1976).

The amount of STB can be measured by determining disease incidence, disease severity, and pycnidia density (Madden et al. 2007, Shaner and Finney 1982). Disease incidence is the proportion of plant units diseased (Madden et al. 2007). In the case of STB, the relevant plant units are leaves; hence, we consider STB incidence to be the proportion of wheat leaves diseased. The degree of infection on a leaf is a measure of disease severity. STB severity is typically measured as the percentage of leaf area covered by lesions (PLACL). Mean STB severity in a plot is usually defined as an average value across a random sample that includes both infected and uninfected leaves. In contrast, conditional STB severity is defined as the mean severity in a sample that includes only infected leaves. Following Rosielle (1972), Shaner et al. (1975), and Shaner and Finney 1982), we consider pycnidia density as another important measure of STB. We use the term "disease intensity" as a general term that refers to both disease severity and pycnidia density. Similar to the definition of conditional severity, we define conditional pycnidia density as the average pycnidia density in a sample of infected leaves. For this study, we collected and analyzed only infected leaves and, therefore, we did not measure STB incidence. The measures of STB that we report here represent conditional severity and conditional pycnidia density.

Earlier studies of STB resistance combined disease severity and incidence using visual assessments based on categorical scales. In studies of Rosielle (1972), Shaner et al. (1975) and Eyal (1992), these scales included both the degree of lesion coverage and the density of pycnidia in lesions; however, in the studies of Brown et al. (2001) and Chartrain et al. (2004), the disease scores were based on leaf coverage by lesions bearing pycnidia (i.e., using a measurement of presence or absence of pycnidia to define STB lesions). The accuracy of all these methods is limited by an inherent subjective bias and a small number of qualitative categories, which may limit success in breeding for STB resistance.

In several studies, the importance of resistance that suppresses pathogen reproduction (i.e., production of pycnidia) was recognized based on qualitative observations of pycnidia coverage (Rosielle 1972; Shaner and Finney 1982; Shaner et al. 1975). Because manual counting of pycnidia is extremely labor intensive, it was only feasible to count pycnidia on a very small scale (Shaner et al. 1975) before the development of new technology to automate this process. Automated image analysis (AIA) provides a promising tool for measuring quantitative disease resistance in the field (Mahlein 2016; Simko et al. 2017). Mutka and Bart (2015) and Mahlein (2016) highlight the importance of standardized imaging methods for reproducibility. We used a novel phenotyping method based on automated analysis of scanned leaf images (Stewart and McDonald 2014; Stewart et al. 2016) in a winter wheat panel planted to 335 elite European cultivars in a replicated field experiment (Kirchgessner et al. 2017). This method benefits from a well-defined procedure of detaching leaves and scanning them under standardized conditions, thus leading to objective and reproducible results. Additionally, it enables generation of large amounts of reliable data at a relatively low cost.

Importantly, our AIA method allowed us to quantitatively separate the net effects of resistance components affecting host damage from resistance components affecting pathogen reproduction. Components of resistance (Parlevliet 1979) are defined as resistance factors suppressing individual processes of the infection cycle (Willocquet et al. 2017). Components of resistance that suppress infection efficiency and lesion expansion are responsible for a reduction in host damage, while components of resistance that suppress spore production (Parlevliet 1979) or pycnidia coverage (Simon and Cordo 1998) are responsible for a reduction in pathogen reproduction. Pathogen reproduction was quantified by automatic counting of asexual fruiting bodies of the pathogen (pycnidia) on wheat leaves (Stewart and McDonald 2014; Stewart et al. 2016) and host damage was measured by automatic detection of discolored leaf areas caused by STB infection.

In this large-scale field experiment, leaves were infected naturally by a genetically diverse local population of $Z$. tritici and the epidemic was allowed to develop naturally. Despite three fungicide treatments that included five active ingredients that eliminated virtually all other diseases, STB infection was widespread across the field experiment. This pervasive natural infection by a fungicide-resistant population allowed us to investigate quantitative resistance in a nearly pure culture of $Z$. tritici under the high-input field conditions typical of Europe. The combination of wet and cool weather conditions favoring development of STB, a large number of wheat cultivars planted in a single location, and utilization of a novel AIA method enabled a comprehensive characterization of quantitative resistance that led to a clear ranking of STB resistance in a broad collection of European winter wheat cultivars.

We report separate rankings of wheat cultivars based on two different components of epidemic outcome, one measured as host damage and the other as pathogen reproduction. We found that the two rankings are considerably different. We identified a phenotypic quantity that combines these two components and found that it correlates best with the ranking based on traditional visual assessments. In this way, we identified new, broadly active sources of resistance to STB in existing European wheat cultivars. Our findings open several possibilities for further genetic studies of quantitative resistance to STB.

\section{MATERIALS AND METHODS}

Plant materials and experimental design. In total, 335 elite European winter wheat (Triticum aestivum) varieties from the GABI-wheat panel (Kollers et al. 2013a,b) were evaluated in this experiment. Two biological replicates of the wheat panel were grown during the 2015-16 growing season in two complete blocks separated by approximately $100 \mathrm{~m}$ at the Field Phenotyping Platform site of the Eschikon Field Station of the ETH Zurich, Switzerland (coordinates $47.449^{\circ} \mathrm{N}, 8.682^{\circ} \mathrm{E}$ ) (Kirchgessner et al. 2017). The complete blocks were composed of 18 rows and 20 
columns consisting of 1.2-by-1.7-m plots, with the genotypes arranged randomly within each block, except for a check variety $(\mathrm{CH}$ Claro) that was planted at least once within each row and column, leading to 21 replicates of $\mathrm{CH}$ Claro within each block. All plots were sown on 13 October 2015.

Practices recommended for conventional, high-input wheat production in Switzerland include applications of fertilizers and pesticides. Fertilizers were applied five times during spring 2016, including boron with ammonium nitrate (nitrogen $52 \mathrm{~kg} / \mathrm{ha}$ ) on 4 March, $\mathrm{P}_{2} \mathrm{O}_{5}$ at $92 \mathrm{~kg} / \mathrm{ha}$ on $7 \mathrm{March}, \mathrm{K}_{2} \mathrm{O}$ at $120 \mathrm{~kg} / \mathrm{ha}$ on $10 \mathrm{March}$, and magnesium with ammonium nitrate on 12 April (magnesium at $15 \mathrm{~kg} / \mathrm{ha}$ and nitrogen at $72 \mathrm{~kg} / \mathrm{ha}$ ) and 20 May (magnesium at $4 \mathrm{~kg} / \mathrm{ha}$ and nitrogen at $19 \mathrm{~kg} / \mathrm{ha}$ ). The preemergence herbicide Herold SC (Bayer) was applied on 29 October 2015 (dose 0.6 liter/ha). The stem shortener Moddus (Syngenta) was applied on 6 April 2016 (dose 0.4 liter/ha) at growth stage (GS) 31 that corresponds to stem elongation (Zadoks et al. 1974). Insecticide Biscaya (Bayer) was applied on 25 May 2016 (dose 0.3 liter/ha) at GS 51 that corresponds to inflorescence emergence.

Fungicides were applied three times: (i) 6 April 2016, Input, Bayer (a mixture of the active ingredients spiroxamin at $300 \mathrm{~g} /$ liter and prothioconazole at $150 \mathrm{~g} /$ liter, dose 1.25 liters/ha, GS 31); (ii) 25 May, Aviator Xpro, Bayer (a mixture of bixafen at $75 \mathrm{~g} /$ liter and prothioconazole at $150 \mathrm{~g} /$ liter, dose 1.25 liters/ha, GS 51); and (iii) 6 June, Osiris, BASF (a mixture of epoxiconazole at $56.25 \mathrm{~g} / \mathrm{liter}$ and metconazole at $41.25 \mathrm{~g} / \mathrm{liter}$, dose 2.5 liters/ha, GS 65 that corresponds to anthesis). In total, the three fungicide applications included five active ingredients representing three modes of action. In particular, bixafen is a succinate dehydrogenase inhibitor; prothioconazole, epoxiconazole, and metconazole are azoles, the main chemical group in the sterol 14a-demethylation inhibitor class of fungicides; and spiroxamin belongs to the morpholine class of fungicides. This strategy of fungicide application aimed to minimize the overall levels of the most common fungal diseases on Swiss wheat.

STB inoculum and calculation of number of cycles of infection. All STB infection was natural, with the majority of primary inoculum likely originating from airborne ascospores coming from nearby wheat fields that surround the Eschikon field site. We obtained rough estimates of the average number of asexual cycles of reproduction within the pathogen population. For this purpose, we used the data from Shaw (1990) showing the effect of temperature on latent period and local weather data coming from the nearby Lindau weather station (Fig. A1; see Appendix, "Estimation of the number of pathogen generations", for details of estimation).

Disease assessment based on automated image analysis. Leaves exhibiting obvious STB lesions were collected two times during the growing season. The first collection was made on 20 May $2016\left(t_{1}\right.$, approximately GS 41 [booting] ) and the second collection was made on 4 July 2016 ( $t_{2}$, approximate range of GS 75 [milk development] to GS 85 [dough development]). For both collections, 16 infected leaves were collected at random from each plot. At $t_{1}$, leaves were collected from the highest infected leaf layer, which was typically the third or fourth fully extended but nonsenescent leaf still visible when counting from the ground or one to three leaf layers below the top leaf. At $t_{2}$, the leaf layer below the flag leaf (F-1) was sampled in each plot. The sampled leaves were placed in paper envelopes, kept on ice in the field, and stored at $4^{\circ} \mathrm{C}$ for 2 days before mounting on A4 paper with printed reference marks and sample names, as described by Stewart et al. (2016). Absorbent paper was placed between each sheet of eight mounted leaves and sheets were pressed with approximately $5 \mathrm{~kg}$ at $4^{\circ} \mathrm{C}$ for 2 to 3 days prior to scanning at 1,200 dpi with a Canon CanoScan LiDE 220 flatbed scanner. The resulting scans were saved as "jpeg" images.

Scanned images were analyzed with the software ImageJ (Schindelin et al. 2015) using a modification of the macro described by Stewart and McDonald (2014) and Stewart et al. (2016) (source code of the macro and a user manual are given in Supplementary File S5). The parameters used for the macro are given in Supplementary Table S1 and an explanation of their meaning is provided in the macro instructions. Figure 1 illustrates the workflow associated with the macro. The maximum length of the scanned area for each leaf was $17 \mathrm{~cm}$. When leaves were longer than $17 \mathrm{~cm}$, bases of the leaves were placed within the scanned area, while the leaf tips extended outside the scanned area. For each leaf, the following quantities were automatically recorded from the scanned image: total leaf area, necrotic and chlorotic leaf area, number of pycnidia, and their positions on the leaf. Necrotic and chlorotic leaf areas were detected based on discoloration of the leaf surface and were not based on the presence of pycnidia. From these measurements, we calculated the PLACL, the density of pycnidia per unit lesion area $\left(\rho_{\text {lesion }}\right)$, and the density of pycnidia per unit leaf area $\left(\rho_{\text {leaf }}\right)$ (Table 1).

The three quantities PLACL, $\rho_{\text {lesion }}$, and $\rho_{\text {leaf }}$ quantify different aspects of conditional intensity of STB in each plot. Although we aimed to collect only infected leaves, there were a few cases in plots with very little STB when the collected leaves did not have necrotic lesions or did not have pycnidia. These leaves were not considered when calculating the mean or median values used for ranking cultivars or assessing the magnitude of effects.

We identified several cases of biased leaf collection at $t_{1}$, where leaves were sampled from lower leaf layers in which some of the leaves exhibited natural senescence, leading to extensive chlorosis and some necrosis. We removed several sheets that were strongly affected by collector bias or natural senescence. Next, we estimated the proportion of remaining scoring errors due to collector bias as $p_{\mathrm{cb}}=0.055 \pm 0.003$ by testing 200 leaves sampled randomly from the entire leaf population.

We found that the original image analysis macro reported by Stewart et al. (2016) identified many falsely detected pycnidia, especially in cultivars with large numbers of thick trichomes. We addressed this issue by improving the macro and performing an extensive optimization of the macro parameters. We changed the macro code to improve detection of pycnidia by enhancing the contrast and optimizing the proportion of red, green, and blue channels in the leaf images. To find an optimal set of macro parameters, we tested more than 160 different sets of parameters on 16 leaves that represented a wide range of numbers of pycnidia per leaf. We chose the parameter set that had the highest concordance coefficient between the numbers of pycnidia per leaf detected by the macro and the numbers of pycnidia counted manually. We then analyzed the entire set of images using this optimized parameter set and tested the outcome in several ways.

First, we visually inspected 209 leaves chosen using random stratified sampling within the range $\rho_{\text {lesion }}<50$. We focused on this range because we found it to be most prone to false detection of pycnidia. For each leaf subjected to visual inspection, we performed a binary (yes or no) assessment of whether the macro correctly detected most of the lesions and pycnidia. This was done by comparing scanned leaf images with overlay images in which the detected lesions and pycnidia were marked by the macro (examples of overlays are shown in Figure 1). Errors that led to incorrect quantification of disease symptoms could be divided into four categories: defects on leaves, collector bias, scanning errors, and deficiencies in the image analysis macro. Leaf defects included insect damage, mechanical damage, insect bodies and frass, other fungi, uneven leaf surfaces creating shadows, and dust particles on leaves. Scanning errors included shadows on leaf edges and folded leaves that resulted from leaves that were not properly flattened prior to scanning. Deficiencies of the macro consisted of recognizing green parts of leaves as lesions, or recognizing dark spaces between light-colored leaf hairs and parts of dark borders around lesions as pycnidia. In total, 21 leaves were deemed to exhibit scoring errors and were removed from the dataset as a result of this procedure. Based on this outcome, we estimated the proportion of 
scoring errors as $p_{\text {tot }}=0.10 \pm 0.02$, out of which the proportion of errors due to false pycnidia was estimated as $p_{\mathrm{fp}}=0.04 \pm 0.03$. Here, the uncertainties are reported in the form of the $95 \%$ confidence intervals $(\mathrm{CI})$ calculated according to $\mathrm{CI}=1.96 \sqrt{ }(p(1-p) / n)$, where $p$ is the sample proportion and $n$ is the sample size.

To further quantify the accuracy of pycnidia detection by the macro, we compared pycnidia counts by the macro to manual pycnidia counts. For this purpose, we selected 30 random leaves from the entire dataset as well as 6 leaves with more than 400 counted pycnidia. We found that pycnidia counts by the macro had high concordance (concordance coefficient $\left[r_{\mathrm{c}}\right]=0.97$ ) and high correlation $\left(r_{\mathrm{s}}=0.96, P=5 \times 10^{-17}\right)$ with manual pycnidia counts. The macro underestimated pycnidia counts, on average, by $26 \pm$ $10 \%$ (the uncertainty here represents the $95 \% \mathrm{CI}$ ).

Disease assessment based on visual scoring. Visual assessments of STB were performed at three time points: 20 May (approximately GS 41), 21 June (approximately GS 75), and 29 June (approximately GS 80). The STB level in each plot was scored by a global assessment of the three uppermost leaf layers on a 1-to-9 scale (1 means no disease to 9 means complete infection) (more details of the scale shown in Supplementary Table S2) based on both STB incidence and severity (Michel 2001). The presence of pycnidia was used as an indicator of STB infection. The absence of pycnidia was interpreted as an absence of STB, even if necrotic lesions were visible. During visual scoring, the presence of other diseases (such as stripe rust, Septoria nodorum blotch, and Fusarium head blight) was assessed qualitatively. All plots were scored with approximately equal time spent on each plot.

Statistical analysis. We compared differences in STB resistance among cultivars for each dataset by pooling together the data points from individual leaves belonging to different replicates and sampling dates. The data from the automated image analysis consisted of approximately 60 data points per cultivar representing the two time points and the two biological replicates (blocks). Cultivar CH Claro was an exception because it was replicated 42 times and, thus, had approximately 1,300 data points from leaf image analysis and 124 data points from visual scoring. The relative

TABLE 1. Important Septoria tritici blotch disease properties determined using automated image analysis

\begin{tabular}{lll}
\hline Quantity & \multicolumn{1}{c}{ Description } & \multicolumn{1}{c}{ Dimension } \\
\hline PLACL & $\begin{array}{c}\text { Percentage of leaf area covered by } \\
\text { lesions }\end{array}$ & Percent \\
$\rho_{\text {lesion }}$ & $\begin{array}{c}\text { Density of pycnidia per unit lesion } \\
\text { area } \\
\text { Density of pycnidia per unit total leaf } \\
\rho_{\text {leaf }}\end{array}$ & $\begin{array}{c}\text { Number of pycnidia/ } / \mathrm{cm}^{2} \\
\text { of lesion } \\
\text { Number of pycnidia } / \mathrm{cm}^{2} \\
\text { of leaf }\end{array}$ \\
\hline
\end{tabular}

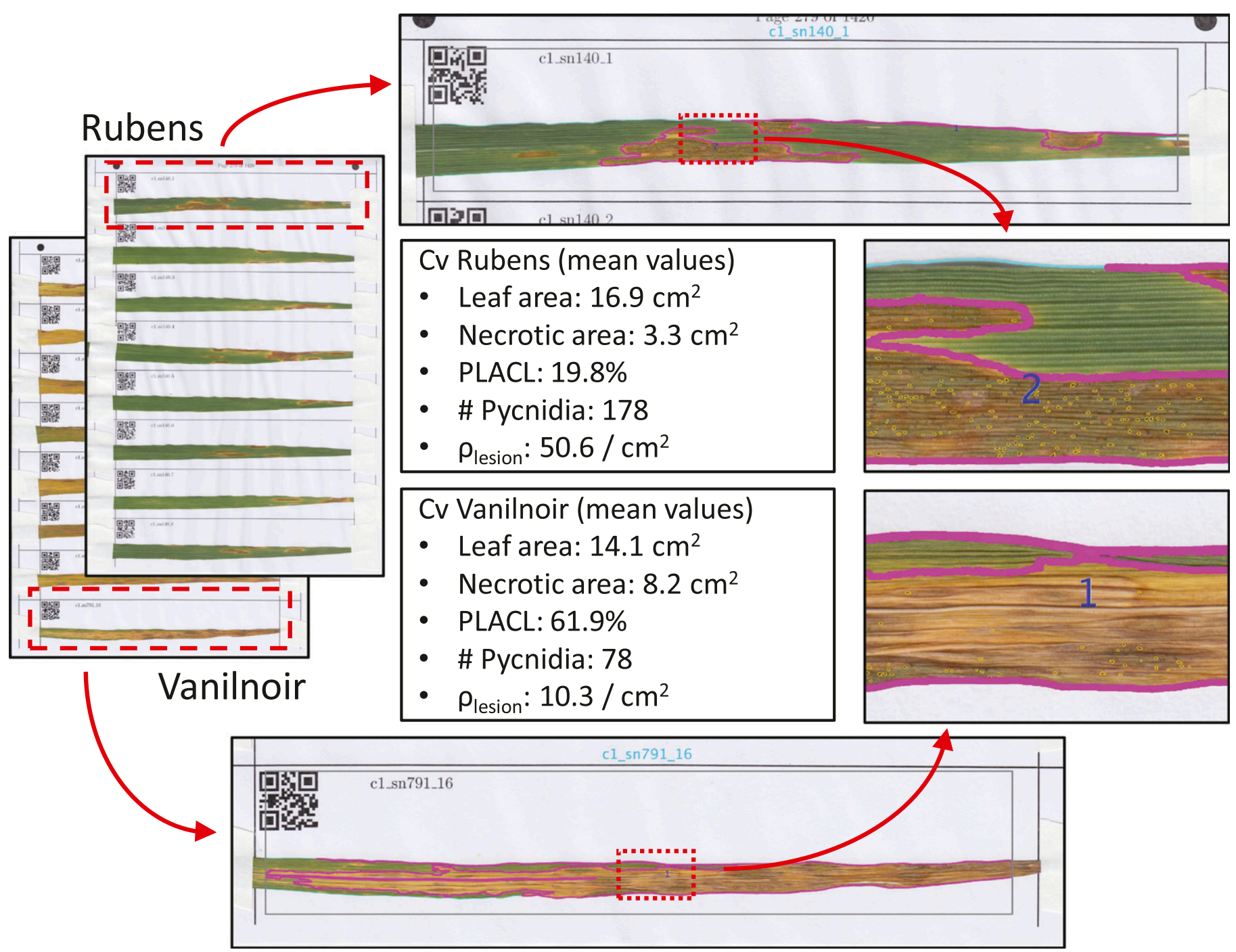

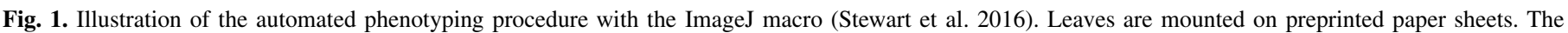

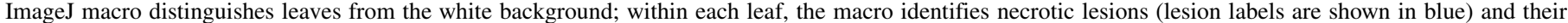
areas; within each lesion, the macro identifies fungal fruiting bodies called pycnidia (black dots). 
STB resistance of all wheat cultivars was ranked based on the means of the conditional measurements of disease intensity (PLACL, $\rho_{\text {lesion }}$, and $\rho_{\text {leaf }}$ ) over approximately 60 individual leaf data points. We also calculated medians and standard errors of the means for each cultivar. Resistance that affects STB incidence was not measured using AIA, because only diseased leaves were analyzed. The visual scoring data were based on three time points and two biological replicates, generating approximately six data points per cultivar. For each cultivar, the area under disease progress curve (AUDPC) was calculated by taking averages of visual scores over the two replicates. It was assumed that infection started from zero at 14 days before the first assessment. To analyze differences between cultivars, these scores were weighted with coefficients that depend on times of assessments, such that each weighted score gives a proportional contribution to the total AUDPC and the average over scores from different replicates and time points gives the total AUDPC (see Appendix, "Calculation of AUDPC based on visual assessments", for details on calculation of AUDPC and weighting of scores). AUDPC was used to rank the cultivars according to visual scoring.

The significance of differences in resistance between cultivars was tested with the global Kruskal-Wallis test (Sokal and Rohlf 2012) using the kruskal.test function in $R$ ( $R$ Core Team 2016) for each data set. For resistance measures showing global differences between cultivars, we determined significantly different groups of cultivars based on pairwise comparisons, whereby any two cultivars in the same group were not significantly different from each other and any two cultivars from different groups were different. Pairwise differences were tested with multiple pairwise Kruskal-Wallis tests using the function kruskal in the package agricolae in $\mathrm{R}$ (de Mendiburu 2016) using the false discovery rate (FDR) of 0.05 for significance level correction (Benjamini and Hochberg 1995) for multiple comparisons.

We determined correlations between cultivar rankings based on AUDPC and using both means and medians of PLACL, $\rho_{\text {lesion }}$, and $\rho_{\text {leaf }}$ for each cultivar. We also computed correlations with respect to means of PLACL, $\rho_{\text {lesion }}$, and $\rho_{\text {leaf }}$ between $t_{1}$ and $t_{2}$ to determine the predictive power of these quantities. In this case, means were taken over approximately 15 leaves originating from the same plot. All correlations were calculated with Spearman's correlation test (Sokal and Rohlf 2012) using the open-source scipy package (https://www. scipy.org) written for the Python programming language (https:// www.python.org).

We analyzed differences between $t_{1}$ and $t_{2}$ in terms of PLACL, $\rho_{\text {lesion }}$, and $\rho_{\text {leaf }}$ to identify cultivars whose resistance increased over time. For this purpose, we used the Wilcoxon rank sum test with the FDR correction for multiple comparisons at $P<0.01$.

Workflow. For clarity, we briefly summarize the workflow of the experiment as a linear sequence of steps. We first collected leaf samples, mounted them on sheets of paper, and scanned them. Next, we performed the basic image analysis using the ImageJ macro, as illustrated in Figure 1. After that, we tested the data and estimated scoring errors using visual examination of leaf images. Finally, we performed detailed statistical analyses using the verified datasets.

\section{RESULTS}

Overall description of the STB epidemic. Despite three fungicide applications with five active ingredients and three modes of action, we observed widespread STB in nearly all of the experimental plots. There were obvious differences in overall levels of STB infection on different cultivars. Comparison of overall levels of STB disease with nearby untreated plots showed that the fungicides significantly suppressed STB development. STB was the dominating disease in the fungicide-treated plots; other leaf diseases were present at very low levels or entirely absent as a result of the fungicide treatments. Hence, this experiment provided an unusual opportunity to assess quantitative STB resistance to infection by a natural, genetically diverse population of $Z$. tritici under conducive field conditions and in the absence of competing wheat diseases.

According to weather data collected from the Lindau weather station located approximately $200 \mathrm{~m}$ away from the field site, the weather in spring and summer 2016 was cool and rainy, conditions highly conducive to development of STB (Appendix, Fig. A2). Average daily temperature between 1 March and 27 July was $12.5^{\circ} \mathrm{C}$ and the total amount of rainfall was $1,245 \mathrm{~mm}$. Based on daily temperature and rainfall data, we estimate that six asexual generations of $Z$. tritici occurred during this period. Between the two leaf sampling dates $t_{1}$ and $t_{2}$, we estimated two asexual generations (see Appendix for details of estimation).

An overview of the dataset. In total, 21,420 leaves were included in the automated analysis pipeline, with an average of 30 leaves/plot. The total leaf area analyzed was $36.8 \mathrm{~m}^{2}$, of which $11.3 \mathrm{~m}^{2}$ was recognized as damaged by STB. The mean analyzed area of an individual leaf was $17 \mathrm{~cm}^{2}$. In total, 2.7 million pycnidia were counted. The mean number of pycnidia within a leaf was 127 . A more detailed description of the overall dataset is given in Table 2. The full dataset can be accessed from the Dryad Digital Repository: https://doi.org/10.5061/dryad.171q4. Correlations between the two biological replicates ranged from 0.23 to 0.66 , with $P$ values ranging from $10^{-4}$ to $10^{-35}$ (Fig. A3; see Appendix, "Correlation between replicates", for more details).

The distributions of the raw data points corresponding to individual leaves with respect to PLACL, $\rho_{\text {lesion }}$, and $\rho_{\text {leaf }}$ are shown in Figures 2 and 3 . The distributions of PLACL, $\rho_{\text {lesion, }}$, and $\rho_{\text {leaf }}$ were nonnormal and had outliers. All of these distributions were continuous, consistent with previous studies that hypothesized that the majority of STB resistance in wheat is quantitative (Stewart et al. 2016).

For the visual assessments conducted across two replicates and three time points, the lowest score was 1 and the highest score was 4 (on a 1-to-9 scale). The lowest value of the AUDPC was 81, the highest value was 154, and the average AUDPC across all cultivars was 103. The Spearman's correlation between visual scores of the two replicates was not significant in the first assessment but was significant in the second and third assessments. The visual assessment also found that yellow rust was present in approximately $1 \%$ of plots on 20 May and in approximately $2 \%$ of plots on 21 June 2016; Septoria nodorum blotch was present in only a single plot on 21 June 2016 (approximately $0.1 \%$ of plots); Fusarium head blight was present in approximately $2 \%$ of plots only on 21 June 2016.

Host damage versus pathogen reproduction. From the raw data obtained via AIA, we derived three quantitative resistance measures: PLACL, $\rho_{\text {lesion, }}$ and $\rho_{\text {leaf }}$ PLACL is defined as the necrotic leaf area divided by the total leaf area, $\rho_{\text {lesion }}$ is the total number of pycnidia divided by the necrotic leaf area, and $\rho_{\text {leaf }}$ is the total number of pycnidia divided by the total leaf area. The measure $\rho_{\text {leaf }}$ can also be calculated from the first two factors as follows:

$$
\begin{aligned}
\text { PLACL } \times \rho_{\text {lesion }}= & (\text { necrotic area } / \text { leaf area }) \times \\
& (\text { number of pycnidia } / \text { necrotic area })= \\
& (\text { number of pycnidia } / \text { leaf area })=\rho_{\text {leaf }}
\end{aligned}
$$

PLACL characterizes host damage due to pathogen infection while $\rho_{\text {lesion }}$ characterizes pathogen reproduction on the necrotic leaf tissue; $\rho_{\text {leaf }}$ is the product of these two quantities, combining host damage with pathogen reproduction. Calculation of these three quantities from the AIA data allowed us to differentiate between host damage and pathogen reproduction, and also to combine these two factors into the most integrative measure of disease intensity, providing a comprehensive insight into different components of $\mathrm{STB}$ resistance. Next, we ranked the wheat cultivars with respect to each of these three quantities.

Ranking of cultivars. Resistance ranking of the cultivars was based on the three measures obtained from AIA (PLACL, $\rho_{\text {lesion, }}$ 
and $\rho_{\text {leaf }}$ ) and the AUDPC calculated from visual scoring. For PLACL, $\rho_{\text {lesion, }}$, and $\rho_{\text {leaf }}$, the distributions differed significantly between cultivars. For each of these three measures, the null hypothesis of identical distributions for all cultivars was rejected by a Kruskal-Wallis global comparison, with $P<2.2 \times 10^{-16}$. However, the global Kruskal-Wallis test did not reveal differences between distributions of the weighted visual scores $(P=1)$. Kruskal-Wallis

TABLE 2. Summary of the leaf analysis

\begin{tabular}{lrrrcr}
\hline Quantities & Total & Mean & Median & Maximum & Minimum \\
\hline Measured & & & & & \\
$\quad$ Leaf area $\left(\mathrm{mm}^{2}\right)$ & $36,784,766$ & 1,717 & 1,685 & 3,541 & 103 \\
Necrotic area $\left(\mathrm{mm}^{2}\right)$ & $11,297,774$ & 527 & 419 & 2,466 & 0 \\
$\quad$ Number of pycnidia $^{2,723,207}$ & 127 & 57 & 4,034 & 0 \\
Derived $^{\mathrm{a}}$ & & & & & \\
PLACL & $\ldots$ & 32 & 24 & 99.8 & 0 \\
$\rho_{\text {lesion }}$ & $\ldots$ & 24 & 16 & 256 & 0 \\
$\rho_{\text {leaf }}$ & $\ldots$ & 7 & 3 & 244 & 0 \\
\hline
\end{tabular}

a PLACL $=$ percentage of leaf area covered by lesions, $\rho_{\text {lesion }}=$ density of pycnidia per unit lesion area, and $\rho_{\text {leaf }}=$ density of pycnidia per unit total leaf area. multiple pairwise comparisons identified three significantly different groups of cultivars for PLACL and four significantly different groups of cultivars for $\rho_{\text {lesion }}$ and $\rho_{\text {leaf }}$ (Figs. 2 and 3). Cultivar ranking based on the Kruskal-Wallis test statistic (mean ranks) is highly correlated with cultivar ranking based on the mean and median values associated with each cultivar (Supplementary File S3), indicating that, in the majority of cases, significantly different cultivars also have different means and medians.

There were notable differences between resistance rankings based on PLACL and $\rho_{\text {lesion }}$ (Fig. 2D). Several of the 30 least resistant cultivars based on host damage were ranked among the most resistant cultivars based on pathogen reproduction. Similarly, some of the most resistant cultivars based on host damage were among the least resistant cultivars based on pathogen reproduction. For example, cultivar Vanilnoir showed high PLACL and low $\rho_{\text {lesion }}$ whereas cultivar Rubens exhibited the opposite pattern. Visual examination of leaves belonging to cultivars that exhibited the largest difference in their ranking between PLACL and $\rho_{\text {lesion }}$ confirmed these patterns. There were relatively low correlations between PLACL and $\rho_{\text {lesion }}$ with respect to means $\left(r_{\mathrm{s}}=0.17, P=\right.$ 0.0022 ) (Fig. 2G) and medians $\left(r_{\mathrm{s}}=0.26, P<10^{-6}\right)$ (Fig. 2F) taken over leaves belonging to the same cultivar. The correlation between
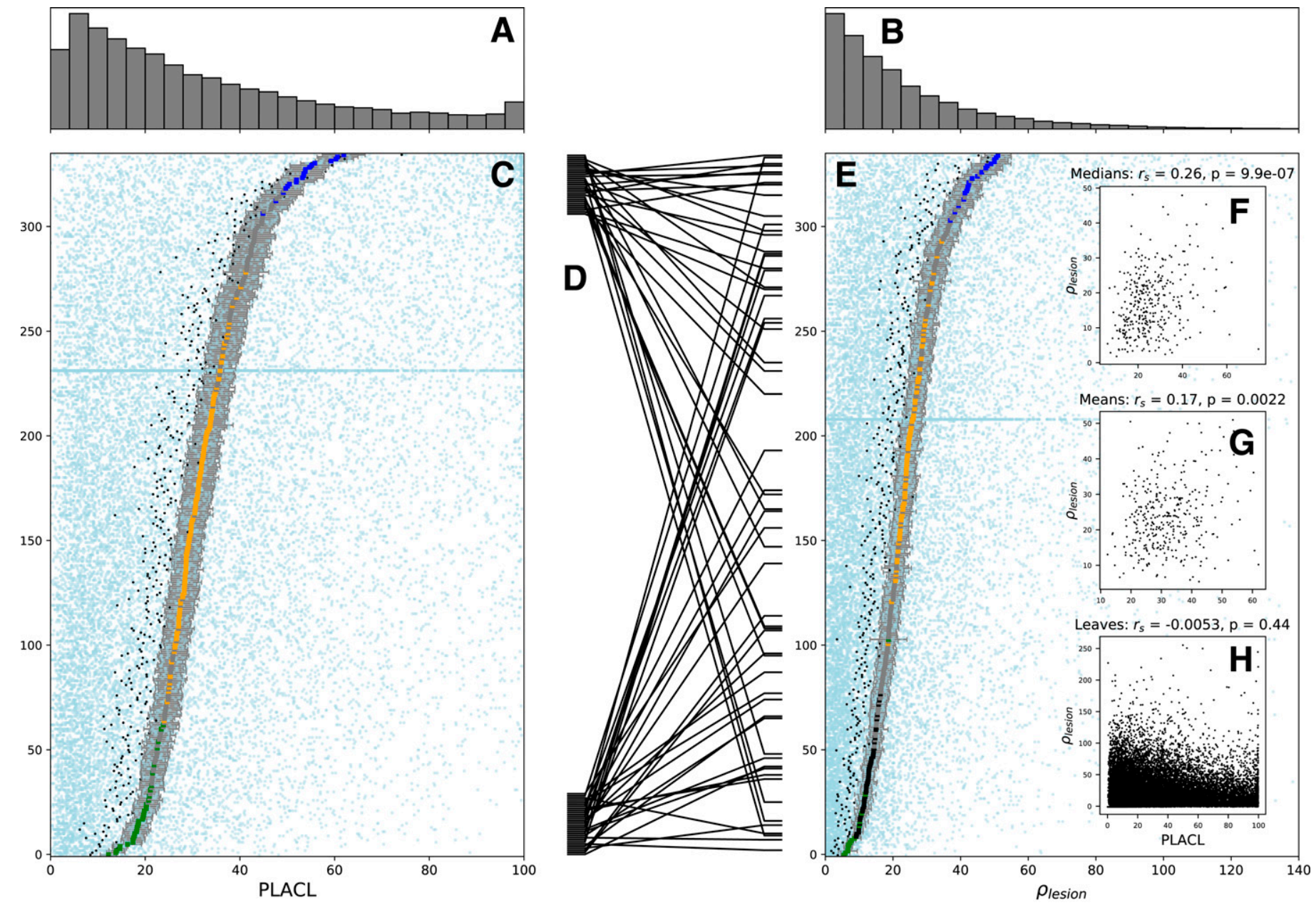

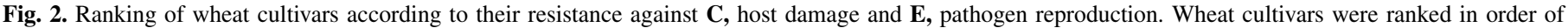

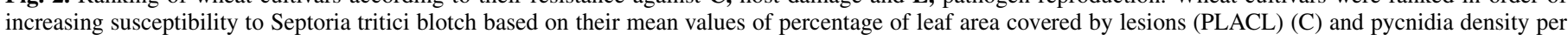

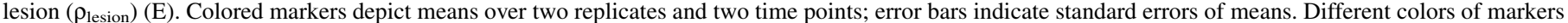

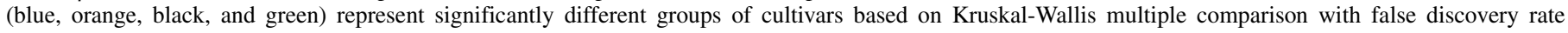

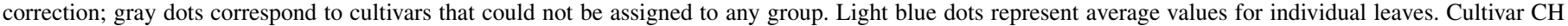

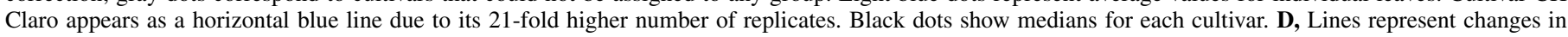

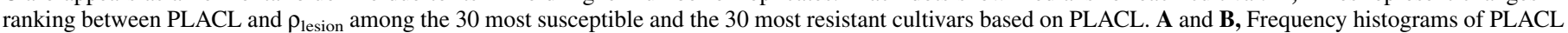

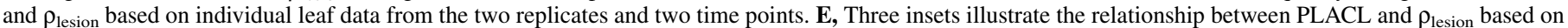
$\mathbf{H}$, individual leaf data; G, means; and F, medians. B and E extend only up to 140 along the $x$-axis, missing 90 data points with values between 140 and 256. 
PLACL and $\rho_{\text {lesion }}$ was not significant when calculated based on individual leaf data pooled together (Fig. 2H). Means, standard errors of means, medians, and Kruskal-Wallis test statistics based on PLACL and $\rho_{\text {lesion }}$ for all cultivars are shown in Supplementary Tables S3, S4, S5, and S6, supporting Figure 2. A brief description of all supplementary files and tables is given in Supplementary File S1.

Automated measures of quantitative STB resistance correlated strongly with the traditional measurement based on AUDPC of visual scores. Medians of PLACL and $\rho_{\text {lesion }}$ correlated significantly $(P<$ $\left.10^{-12}\right)$ with the AUDPC $\left(r_{s}=0.38\right.$ and 0.53 , respectively $)$. Correlations between AUDPC and means were somewhat weaker but also significant. The strongest correlation was between the combined measure $\rho_{\text {leaf }}$ and the AUDPC (means: $r_{s}=0.63$ and medians: $r_{s}=0.58$ ) (Fig. 3 ). More figures of cultivar ranking that support Figures 2 and 3 with different combinations of measures are shown in Supplementary File S2. Means, standard errors of means, medians, and Kruskal-Wallis test statistics based on $\rho_{\text {leaf }}$ for all cultivars are shown in Supplementary Tables S7 and S8, supporting Figure 3.

Predictors of epidemic development. Thus far, we have analyzed data for each cultivar based on pooling the sampling dates $t_{1}$ and $t_{2}$. Next, we considered data from the sampling dates $t_{1}$ and $t_{2}$ separately. An important question is: To what extent can we predict a measure of disease at $t_{2}$ from measurements made at $t_{1}$ ? We addressed this question by investigating correlations between $t_{1}$ and $t_{2}$ with respect to each of the three measures: PLACL, $\rho_{\text {lesion, }}$, and $\rho_{\text {leaf }}$ (Fig. 4). A higher degree of correlation corresponds to a higher predictive power. Consider the first column in Figure 4 that illustrates how PLACL in $t_{1}$ correlates with PLACL, $\rho_{\text {lesion, }}$, and $\rho_{\text {leaf }}$ in $t_{2}$. PLACL in $t_{1}$ correlates somewhat better with $\rho_{\text {lesion }}$ in $t_{2}$ than with $\rho_{\text {leaf }}$ or PLACL in $t_{2}$. However, PLACL in $t_{1}$ is a poorer predictor for the three quantities in $t_{2}$ than the quantities that include pycnidia counts, $\rho_{\text {lesion }}$ and $\rho_{\text {leaf }}$ (Fig. 4 ; compare the first column with the second and third columns). The highest correlations emerged between $\rho_{\text {leaf }}$ in $t_{1}$ and $\rho_{\text {lesion }}$ in $t_{2}\left(r_{\mathrm{s}}=0.44\right)$ and between $\rho_{\text {leaf }}$ in $t_{1}$ and $\rho_{\text {leaf }}$ in $t_{2}\left(r_{\mathrm{s}}=0.50\right)$. The best predictor for PLACL (the measure of host damage that is most likely to reflect decreased yield) in $t_{2}$ is $\rho_{\text {lesion }}$ (the most inclusive measure of pathogen reproduction) in $t_{1}$ (Fig. 4, first row). A general account of the correlations and predictive power among the measured quantities in $t_{1}$ and $t_{2}$ is given in Figure 4. We investigated more subtle patterns of this comparison in Supplementary File S4, where we separated the effect of cultivar differences from the overall effect.

For a subset of 39 cultivars, we performed similar measurements in a preliminary experiment conducted in 2015 (Stewart et al. 2016).
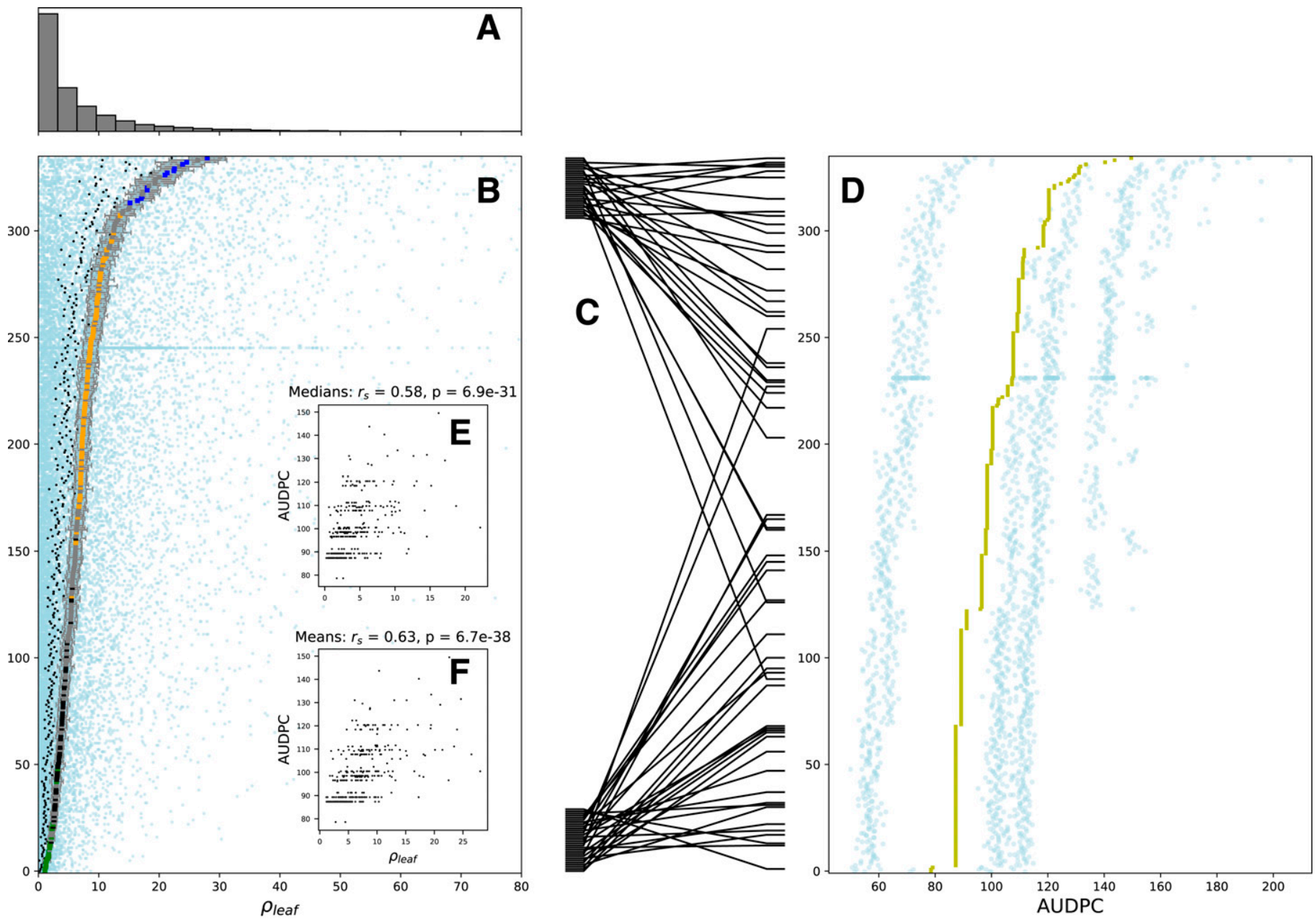

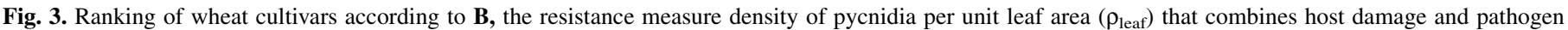

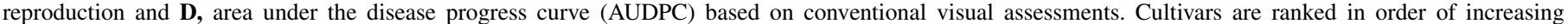

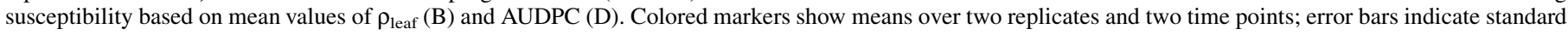

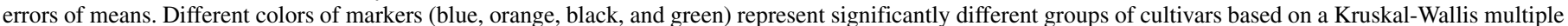

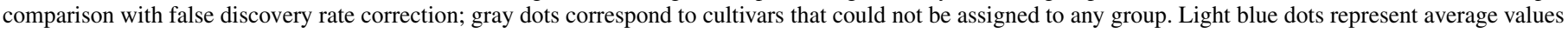

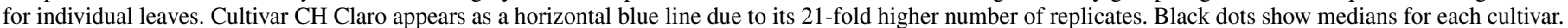

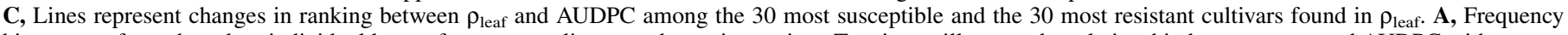

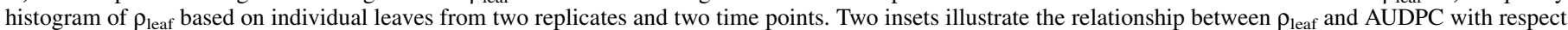

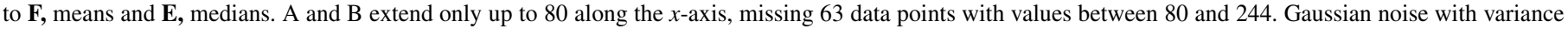
$\sigma^{2}=4$ was added to raw (blue) data points in D to improve their visibility. 
Comparisons between the outcomes in 2016 and 2015 indicated that PLACL exhibited no significant correlation, whereas $\rho_{\text {lesion }}$ and $\rho_{\text {leaf }}$ showed moderate and significant correlations $\left(r_{s}=0.46, P=\right.$ 0.0035 for $\rho_{\text {lesion }}$ and $r_{s}=0.41, P=0.0092$ for $\rho_{\text {leaf }}$ ). This further highlights the importance of measuring quantitative resistance based on pathogen reproduction because it provided a more consistent measure of STB resistance between years and environments than measures of STB resistance based on host damage.

Increase of resistance to STB between $t_{1}$ and $\boldsymbol{t}_{2}$. Next, we investigated the difference between $t_{1}$ and $t_{2}$ to identify cultivars that exhibit an increase in resistance over time. We will refer to this as "late-onset" resistance. On average, each of the quantities PLACL, $\rho_{\text {lesion }}$, and $\rho_{\text {leaf }}$ increased between the two time points (Fig. 5). The difference was somewhat larger for $\rho_{\text {leaf }}$ than for PLACL and $\rho_{\text {lesion }}$. The overall mean differences were smaller than the variance of differences in individual cultivars. This is because positive changes in some cultivars were compensated by negative changes in other cultivars (Fig. 5).

We investigated the negative changes in more detail. We identified 53 cultivars in total (Fig. 5, indicated in red) that exhibited significant negative changes with respect to at least one of the quantities PLACL, $\rho_{\text {lesion}}$, or $\rho_{\text {leaf }}$. The number of cultivars showing significant negative change in PLACL (41 cultivars) was higher than in $\rho_{\text {lesion }}$ (12 cultivars) (Fig. 5D, Venn diagram). Four cultivars showed significant negative change with respect to $\rho_{\text {leaf }}$. The number of cultivars exhibiting significant negative change in
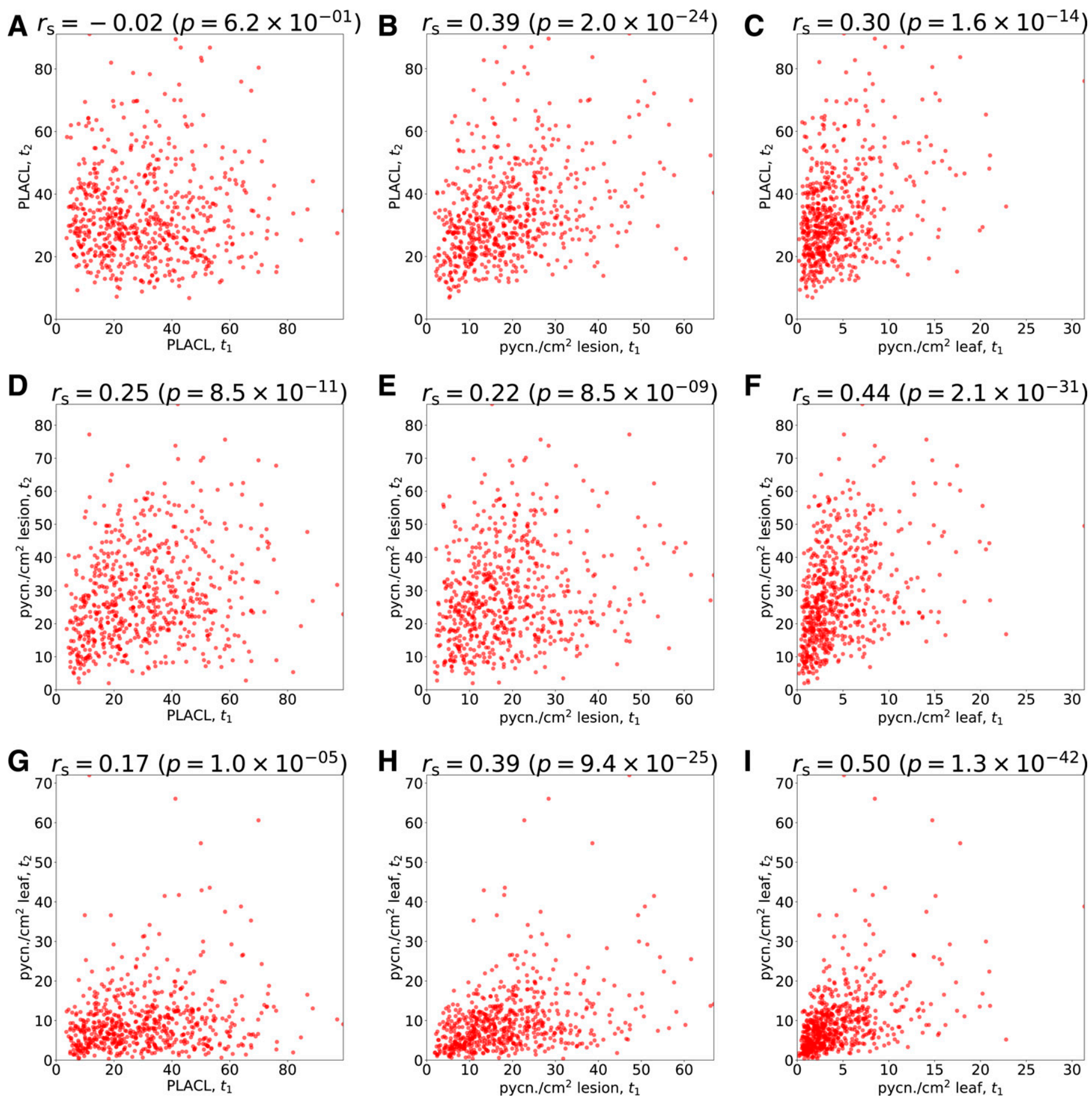

Fig. 4. A to I, Correlations of measures of quantitative Septoria tritici blotch resistance between the first time point $\left(t_{1}\right)$ and the second time point $\left(t_{2}\right)$. The degree of correlation was quantified using Spearman's correlation coefficient $\left(r_{\mathrm{s}}\right)$. Each data point represents an average over approximately 15 leaves within an individual plot. 
terms of only one quantity were 38 for PLACL, 11 for $\rho_{\text {lesion }}$, and 0 for $\rho_{\text {leaf }}$. Interestingly, none of the cultivars exhibited significant negative change for both PLACL and $\rho_{\text {lesion. }}$ PLACL decreased the most in cultivars Achat and Mewa (by 54 and 41 units, respectively), $\rho_{\text {lesion }}$ decreased the most in cultivars Parador and Cetus (by approximately 33 and 26 units, respectively), and $\rho_{\text {leaf }}$ decreased the most in cultivars Cetus and Urban (by 12 units and 1 unit, respectively). Lists of cultivars with their corresponding magnitudes of changes are given in Supplementary Tables S9, S10, and S11.

\section{DISCUSSION}

Novel aspects of the experimental design. Although fungicides suppressed STB development as compared with untreated plots, the most important benefit of the fungicide applications in the context of this experiment was to eliminate competing diseases (rusts, powdery mildew, tan spot, and Septoria nodorum blotch) that usually coexist with STB in naturally infected fields. This resulted in a nearly pure culture of STB across both replications. Virtually every disease lesion found on a leaf was shown to be the result of an infection by Z. tritici. The widespread STB infection found in this experiment could be explained by the cool and wet weather during the 2015-16 growing season that was highly conducive to development of STB, coupled with a significant amount of resistance to azoles in European populations of Z. tritici (Brunner et al. 2008).

This experimental design provided an unusual opportunity to directly compare levels and development of STB infection and STB resistance across a broad cross-section of elite European winter wheat cultivars. Combined with the novel automated image analysis method, this allowed us to collect a large amount of high-quality

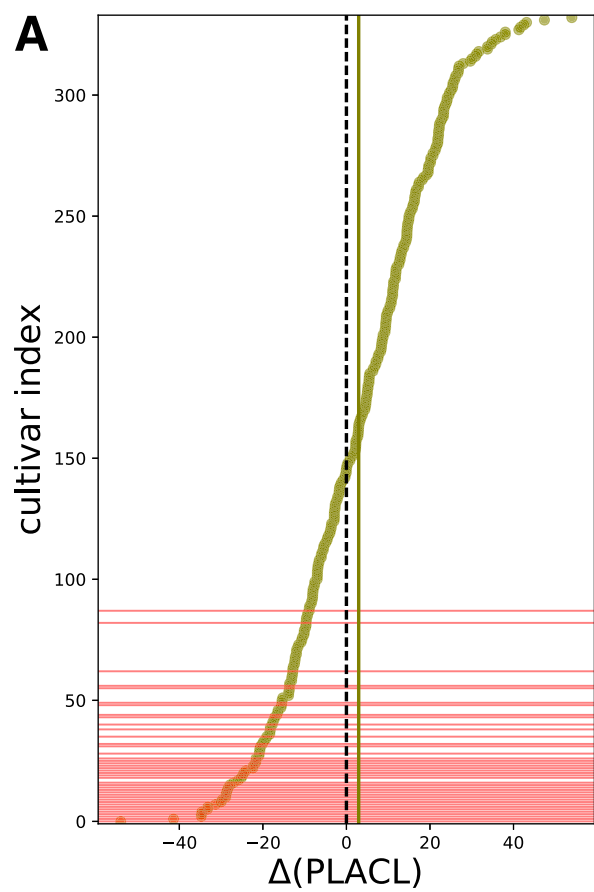

D
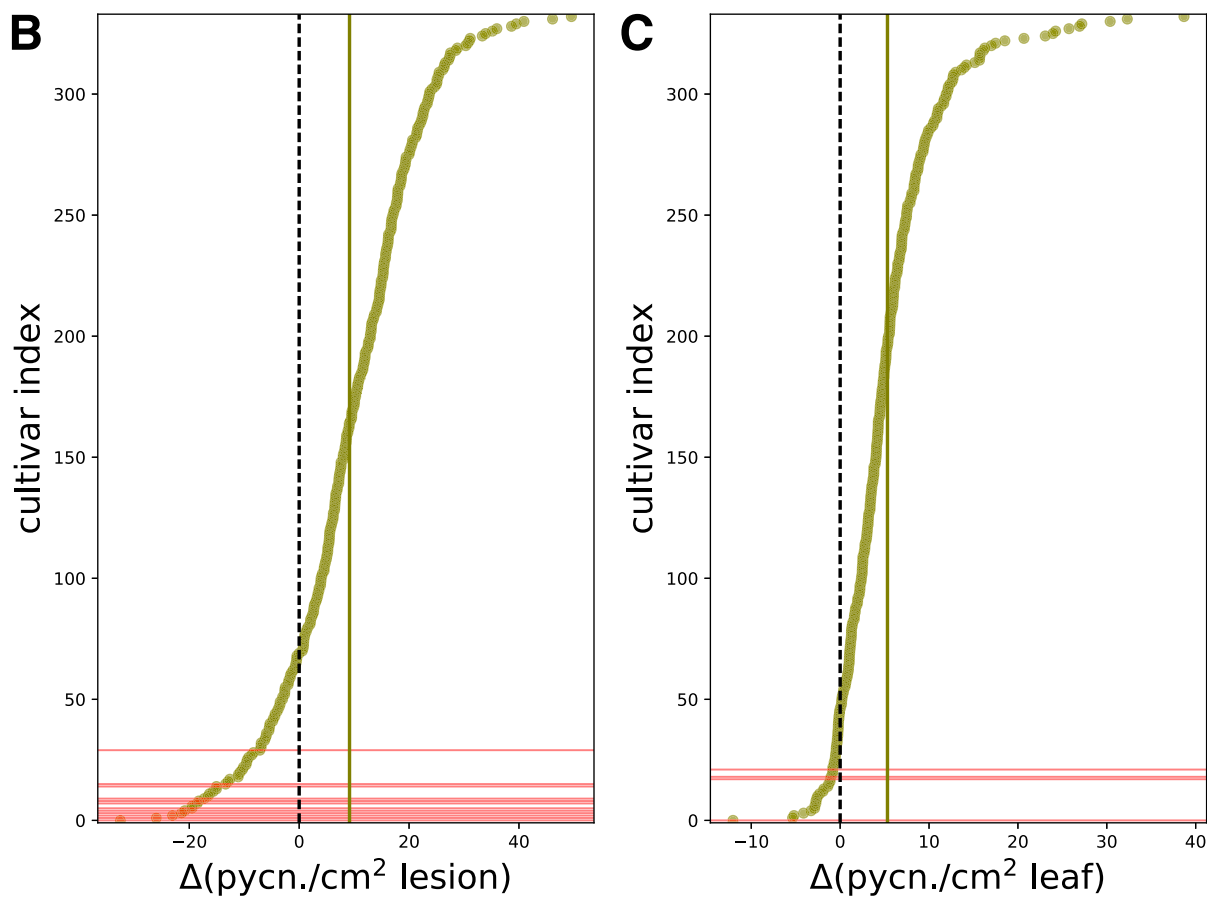

\section{pycn./cm² leaf}

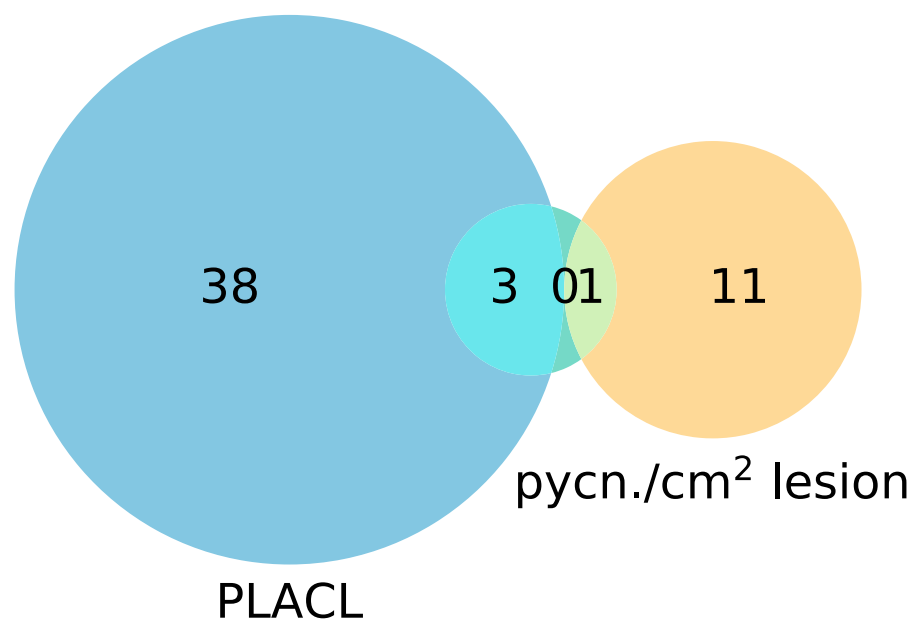

Fig. 5. Indicators of late-onset Septoria tritici blotch resistance. The $x$-axes show differences between mean values at time point $t_{2}$ and time point $t_{1}$ for the three quantities (yellow markers): A, percentage of leaf area covered by lesions (PLACL); B, pycnidia density per lesion $\left(\rho_{\text {lesion }}\right)$; and $\mathbf{C}$, density of pycnidia per unit leaf area $\left(\rho_{\text {leaf }}\right)$. The $y$-axes represent cultivar indices; cultivars are sorted in order of increasing differences. Solid vertical lines show differences averaged over all cultivars; dashed vertical lines show zero differences. Cultivars exhibiting significant negative differences (according to Wilcoxon rank sum test with the false discovery rate correction, $P<0.01)$ are shown in red. $\mathbf{D}$, Venn diagram of cultivars with significant negative differences with respect to the three measures of resistance. 
data with a relatively low workload. The measures of resistance that we characterized here, on average, represent "general" or "field" resistance because the experiment was conducted using natural infection in a year that was highly conducive to STB. Cultivars that were highly resistant under these conditions are more likely to be broadly resistant when exposed to the typical genetically diverse Z. tritici populations found around the world (Linde et al. 2002; Zhan et al. 2003).

Comparison between datasets obtained from automated image analysis and visual scoring. Conventional visual assessment typically quantifies leaf necrosis (host damage) caused by the pathogen by integrating disease severity (as PLACL) and incidence (as the proportion of leaves having necrotic lesions) into a single index. Pycnidia (an indicator of pathogen reproduction) are typically considered as a qualitative trait of presence or absence that helps to separate STB lesions from lesions caused by other leafspotting diseases (e.g., tan spot or Septoria nodorum blotch) occurring on the same plants. The conventional visual assessment is fast: only approximately $9 \mathrm{~h}$ in total were needed to assess more than 700 small plots three times during the season by a single person (i.e., approximately $3 \mathrm{~h}$ at each measurement date).

Visual scoring benefits from a large sample size, because almost all leaves in a plot are considered during a typical visual assessment compared with only 15 leaves used for image analysis. However, due to the subjective nature of the conventional scoring process, the sample size used is not clearly defined and we could not obtain statistically significant differences between cultivars based on the conventional visual scores. Moreover, assessment of changes in trait values between two time points is very difficult with conventional visual scores. Uncertainty in detection of pycnidia in the conventional measurement may lead to misclassification of resistance. The degree of STB resistance may be overestimated on cultivars that are susceptible to host damage (have high PLACL) but suppress pathogen reproduction (have low $\rho_{\text {lesion}}$ ), because failure to detect pycnidia may be interpreted as an absence of the disease. On the other hand, if the visual assessment finds pycnidia (typically scored as present or absent), the resistance of a cultivar may be underestimated if the degree of suppression of pycnidia production is not considered.

In contrast, automated analysis of individual leaves enables independent measurement of different aspects of conditional disease intensity. The advantage of the automated method based on leaf images is that it accounts for both host damage and pathogen reproduction in a reproducible, quantitative way with a well-defined sample size. This alleviates the uncertainty in detecting pycnidia and also allowed us to find statistically significant differences between cultivars. However, this method is more labor intensive than visual scoring. Approximately 360 person-hours were needed to collect and process approximately 22,000 leaves and obtain the raw data.

Although this is an automated method, one needs to carefully determine its error rate at every use (as we described in the Materials and Methods), because errors may be cultivar specific and also depend on environmental conditions. Sources of errors can be minimized in the future by further improving the experimental methodology. For example, leaves with severe defects can be excluded at the stage of mounting on paper sheets, scanning errors can be minimized by introducing an additional control step after scanning, and collector bias can be minimized by improved training of leaf collectors. Importantly, we plan to further minimize current deficiencies in pycnidia and lesion detection by incorporating the image analysis into a machine-learning algorithm.

Manual generation of this large dataset that included more than 2 million pycnidia would not be practical. However, we demonstrated that combining cheap flatbed scanners with public domain software to conduct automated image analysis makes it feasible to separate different components of epidemic outcome affected by presumably different components of quantitative host resistance on a large scale.
Host damage versus pathogen reproduction. Biologically, PLACL reflects the pathogen's ability to invade and damage (necrotize) the host leaf tissue, whereas $\rho_{\text {lesion }}$ reflects the pathogen's ability to convert the damaged host tissue into reproductive structures and eventually into offspring. From the host's perspective, PLACL can be interpreted as the degree of susceptibility to damage caused by the pathogen during the infection process (e.g., through secretion of phytotoxins) or by host defense reactions (e.g., the hypersensitive response) activated after detecting the pathogen. In contrast, $\rho_{\text {lesion }}$ can be interpreted as measuring the host's ability to suppress pathogen reproduction: a more susceptible host enables more pathogen reproduction per unit of infected leaf area. Automated image analysis enabled us to differentiate between host damage and pathogen reproduction and, hence, to measure these as separate components of an STB epidemic that reflects differences in components of resistance between wheat cultivars.

The phenotypic differences observed in our experiment may reflect different sets of genes underlying the two resistance traits. We hypothesize that PLACL reflects the additive actions of toxin sensitivity genes carried by different wheat cultivars that interact with host-specific toxins produced by the pathogen, as shown for Parastagonospora nodorum on wheat (Friesen et al. 2008; Oliver et al. 2012). We hypothesize that pycnidia density reflects additive actions of quantitative resistance genes that recognize pathogen effectors (e.g., AvrStb6 recognized by Stb6) (Zhong et al. 2017) and activate plant defenses in a quantitative way (Krattinger et al. 2009). Another possibility is that pycnidia density reflects the availability of nutrients or the concentrations of antimicrobial compounds present in the necrotic host tissue. We anticipate testing these hypotheses by combining the phenotypic data reported here with wheat genome data (International Wheat Genome Sequencing Consortium 2014) to conduct a genome-wide association study (GWAS) aiming to identify chromosomal regions and candidate genes underlying these components of quantitative STB resistance that may explain the observed differences in epidemics.

The density of pycnidia per unit leaf area $\left(\rho_{\text {leaf }}\right)$ is a measure of disease intensity that incorporates both host damage and pathogen reproduction, reflecting the pathogen's overall ability to convert healthy host tissue into reproductive units that can drive a new cycle of infection. The complex nature of quantitative host-pathogen interactions may lead to high PLACL combined with low $\rho_{\text {lesion }}$ or low PLACL combined with high $\rho_{\text {lesion. }}$. In both of these cases, the infection is less severe than when an interaction leads to a high $\rho_{\text {leaf }}$. Therefore, we believe that $\rho_{\text {leaf }}$ better characterizes overall host resistance, pathogen fitness, and STB intensity than PLACL or $\rho_{\text {lesion. }}$.

Ranking of cultivars. The overall differences among cultivars were relatively small, except for a small number of cultivars at each extreme. These small differences are reflected in the limited number of significantly different groups of cultivars. However, our analyses showed that ranking of cultivars differs considerably with respect to the two measures of quantitative resistance: resistance to host damage and resistance to pathogen reproduction (Fig. 2D). More specifically, we identified cultivars that strongly exhibit one component of epidemic outcome, whereas the other component is virtually absent (Fig. 2F, points in the upper-left and lower-right corners). We expect that resistance that lowers pycnidia production will have a greater overall impact on reducing damaging STB epidemics because it will reduce the rate of epidemic increase more than resistance to host damage. Conventional phenotyping based on visual assessment does not enable separation of these different components of epidemic outcome and the underlying components of resistance.

We expected that conventional visual assessment (based on AUDPC) would correlate best with the measurement of host damage (PLACL), because conventional assessment consists mainly of quantifying leaf necrosis while using pycnidia mainly to confirm the presence of STB. Surprisingly, the AIA measure that combines 
host damage and pathogen reproduction $\left(\rho_{\text {leaf }}\right)$ gave the best correlation with the conventional visual assessment. A possible explanation for this high correlation is that the conventional assessment may actually quantify pycnidia, although in a subjective way. An alternative explanation is that, because conventional assessment includes both disease severity and incidence, it captures the overall pathogen population size that depends on both host damage and pathogen reproduction.

The correlation between our combined measure $\left(\rho_{\text {leaf }}\right)$ and the conventional measure (AUDPC) in Figure 3 indicates that breeders may have selected for cultivars that combine resistance to host damage and resistance to pathogen reproduction. This hypothesis is supported by the small but significant positive correlations between PLACL and $\rho_{\text {lesion }}$ in terms of both means and medians over individual cultivars (Fig. 2F). (See Appendix, "Use of means versus medians in the analysis of STB resistance", for a discussion on the use of means versus medians in the analysis.)

Our rankings are based on conditional measures of disease intensity and do not take into account resistance that might lead to a decreased number of diseased leaves. In order to include this aspect of resistance in future rankings, our conditional measurements will need to be complemented by measurements of STB incidence.

Predictors of epidemic development. PLACL on the upper leaves is a key determinant of disease-induced yield loss (Brokenshire 1976). Hence, the ability to predict PLACL on the upper leaves late in the growing season based on an early-season measurement may improve disease control. Our results suggest this possibility; the measure that quantifies pathogen reproduction, $\rho_{\text {lesion }}$, evaluated early in the season (at $t_{1}$, approximately GS 41) predicts the lateseason host damage (PLACL at $t_{2}$, approximately GS 75 to 85) better than early-season PLACL or $\rho_{\text {leaf }}$ (Fig. 4, compare B with A and $\mathrm{C}$ ). We postulate that this finding could improve decision making for fungicide application; one may need to apply fungicides only if $\rho_{\text {lesion }}$ exceeds a certain threshold early in the season.

Increase of resistance to STB between $\boldsymbol{t}_{\mathbf{1}}$ and $\boldsymbol{t}_{\mathbf{2}}$. Negative changes with respect to traits characterizing host susceptibility (PLACL, $\rho_{\text {lesion }}$, and $\rho_{\text {leaf }}$ ) suggest an increase in host resistance over time. We found that none of the cultivars exhibited this property for both PLACL and $\rho_{\text {lesion. Accordingly, none of the }}$ cultivars showed a significant decrease only for the combined measure $\rho_{\text {leaf }}$. This may indicate that the genetic basis of the lateonset resistance differs for host damage and pathogen reproduction. These outcomes may help to reveal the genetic basis of "late-onset" resistance to STB (e.g., using GWAS or QTL mapping).

Conclusions. We utilized a novel phenotyping technology based on automated analysis of digital leaf images to compare quantitative resistance to STB in 335 European wheat cultivars naturally infected by a highly variable local population of $Z$. tritici. This method allowed us to distinguish between resistance components affecting host damage (PLACL) and resistance components affecting pathogen reproduction $\left(\rho_{\text {lesion }}\right)$. Because measurements of pycnidia density cannot be accomplished on such a large scale with traditional assessment methods, our new method provides a powerful tool for measuring quantitative resistance to STB. As suggested by Simko et al. (2017), digital phenotyping reduces subjectivity in trait quantification and may reveal small but meaningful differences that would not be observed with conventional visual assessment. Further development of this method could involve adjustment of the analysis parameters to be optimized for each cultivar separately, machine learning for more precise detection of symptoms, and combining it with incidence data gathered by image analysis of high-quality canopy images produced by devices like the phenomobile (Deery et al. 2014) or the ETH field phenotyping platform (Kirchgessner et al. 2017).

Outlook. Our approach can be readily applied to classical phenotype-based selection and breeding. Cultivars that show high resistance to both host damage and pathogen reproduction will be most likely to strongly suppress the pathogen population at the field level and result in less overall damage due to STB. Importantly, cultivars that show the highest resistance based on either host damage or pathogen reproduction can be used in breeding programs as independent sources of different components of resistance. We believe our approach provides a powerful method to specifically breed wheat cultivars carrying resistance that suppresses pathogen reproduction.

Although the ability to separate phenotypes associated with two different aspects of resistance provides new avenues for resistance breeding, our hypothesis that the components of resistance explaining the two components of epidemic outcome are under separate genetic control remains to be confirmed by further research. We anticipate that future genetic studies (e.g., using GWAS) based on these phenotypic data will enable us to identify genetic markers that are linked to the different types of resistance. These markers could then enable joint selection of the different forms of resistance via marker-assisted breeding or in a genomic selection pipeline. If we can validate our hypothesis that toxin sensitivity genes underlie differences in PLACL among cultivars, the breeding objective would be to remove these sensitivity genes (Friesen et al. 2008; Oliver et al. 2012).

\section{APPENDIX}

Estimation of the number of pathogen generations. We estimated the number of asexual cycles of pathogen reproduction (number of generations) using data on the dependency of the latent period of Z. tritici on temperature (Shaw 1990, figure 5). We are interested in the overall relationship between the latent period and the temperature and would like to use the largest amount of data available. For this reason, we pooled together the data available for two cultivars, Avalon and Longbow, recorded by Shaw (1990). Next, we fitted the polynomial function

$$
1 / \Delta t_{l}=a \theta-b \theta^{4}
$$

to the resulting data. Here, $\Delta t_{l}$ is the latent period, $\theta$ is the temperature, and $a$ and $b$ are fitting parameters. The outcome is shown in Fig. A1. Best-fit values of parameters are

$$
a=(4.5 \pm 0.6) \times 10^{-3}, b=(3.9 \pm 1.0) \times 10^{-7}
$$

Uncertainties in equation A.2 represent the $95 \%$ CI calculated from standard errors (goodness of fit: $R^{2}=0.7$ and standard error of regression: $\left.s=5.5 \times 10^{-3}\right)$.

We then used average daily temperatures and the amount of rainfall measured at the Lindau weather station located close to our experimental site to estimate the number of pathogen generations $\left(n_{g}\right)$. We performed estimation of $n_{g}$ from the period of most active vertical growth until harvest (1 March until 27 July) and between the two sampling dates (from 20 May until 4 July). First, we determined the average latent period from the daily temperature averaged over the growing season using equation A.1 with the parameter values from equation A.2. This resulted in the value $\Delta t_{l}=21$ days. After that, we introduced a constraint on the number of pathogen generations using the rainfall data. According to our current understanding, rainfall is the most efficient way to release and disperse the asexual pycnidiospores. For this reason, we assumed that a cycle of asexual reproduction could only be completed after a day with at least $5 \mathrm{~mm}$ of rainfall (Zhan et al. 2002). In this way, we estimated an average of approximately six cycles of asexual reproduction between 1 March and 27 July and approximately two cycles between the two sampling dates $t_{1}$ and $t_{2}$ (Fig. A2).

Calculation of AUDPC based on visual assessments. We denote the values of visual scores recorded on the first $\left(\tau_{1}\right)$, second $\left(\tau_{2}\right)$, and third $\left(\tau_{3}\right)$ dates (20 May, 21 June, and 29 June 2016) of 
visual assessments as $A_{1}, A_{2}$, and $A_{3}$. AUDPC was calculated for each cultivar using the visual scores in the following manner:

$$
\text { AUDPC }=1 / 2\left[\tau_{2} A_{1}+\left(\tau_{3}-\tau_{1}\right) A_{2}+\left(\tau_{3}-\tau_{2}\right) A_{3}\right]
$$

where $\tau_{1}=14$ days, $\tau_{2}=44$ days, and $\tau_{3}=52$ days. Here, we assumed that disease started from zero 14 days before the first scoring and values of $A_{1}, A_{2}$, and $A_{3}$ are taken as average scores over the two replicate plots. Equation A.3 uses a trapezoidal function to interpolate between the time points in order to calculate the area under the curve. This assumes that score values are connected by linear segments. Values of AUDPC are shown for each cultivar in Figure 3D.

To analyze differences between cultivars, we weighted scores $A_{1}$, $A_{2}$, and $A_{3}$ in the following manner: $a_{1}=3 \tau_{2} A_{1} / 2, a_{2}=3\left(\tau_{3}-\tau_{1}\right) A_{2} / 2$, and $a 3=3\left(\tau_{3}-\tau_{2}\right) A_{3} / 2$. The coefficients were chosen such that weighted scores $a_{1}, a_{2}$, and $a_{3}$ give proportionate contribution to the AUDPC and the arithmetic average over them gives the AUDPC. The weighted scores from each replicate and each time point were given as a set of points corresponding to each cultivar (total of six measurement points per cultivar). One score was missing for one of the replicates in several cultivars. For these cultivars, we used only

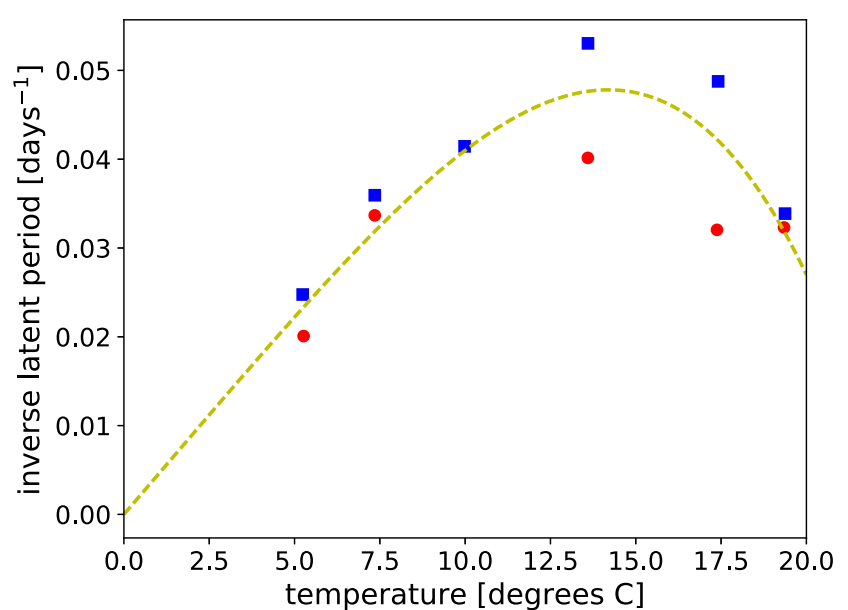

Fig. A1. Inverse of latent period of Zymoseptoria tritici as a function of the temperature based on data from Shaw (1990, figure 5). Data from controlledenvironment experiments for cultivars Longbow (circles) and Avalon (squares). Dashed curve is the best fit using the function in equation A.1.

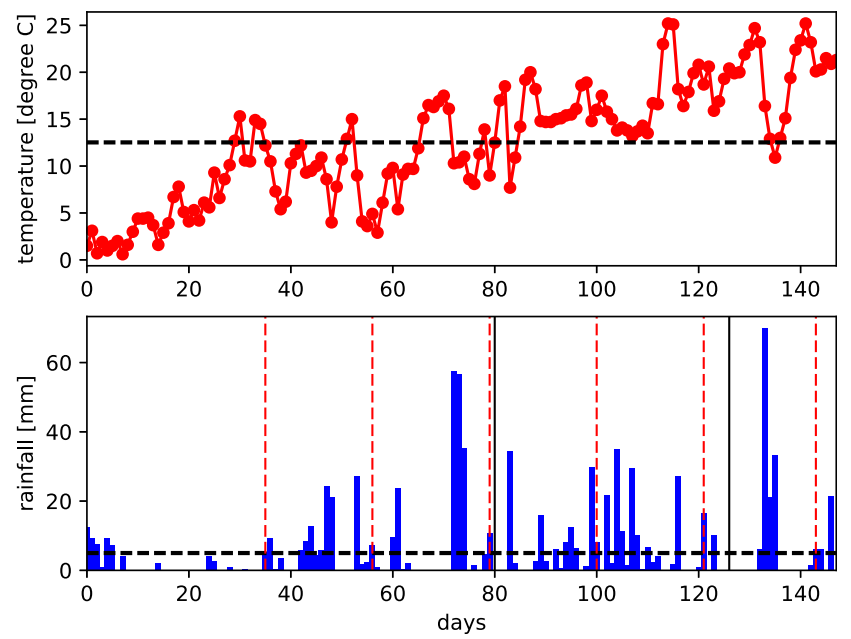

Fig. A2. Temperature and rainfall data recorded at the Lindau weather station (data from http://www.agrometeo.ch/de/meteorology/datas) for the period between 1 March and 27 July 2016. Red vertical lines indicate the estimated generation times and black vertical lines show the sampling dates $t_{1}$ and $t_{2}$. five measurement points for statistical analysis. We also calculated grand means over raw (not weighted) visual scores for each cultivar by taking arithmetic means over measurements in two replicates and three time points (six measurement points). Similar to the case of weighted scores, when scores were missing in one of the replicates at one of the time points, we calculated arithmetic means over the five values that were present.

Statistical differences between cultivars based on visual scoring could, in principle, be tested in a manner similar to those based on image analysis data. We tested differences between distributions based on the weighted visual scores of the cultivars. The global Kruskal-Wallis test (R Core Team 2016) revealed no differences (Kruskal-Wallis $\chi^{2}=194.39$, df $=335, P=1$ ). Surprisingly, when using unweighted visual scores, there were differences between cultivars (Kruskal-Wallis $\chi^{2}=642.4$, df $=335, P<2.2 \times 10^{-16}$ ). Pairwise multiple comparison with an FDR $P$ value correction (Benjamini and Hochberg 1995) found that the 23 most susceptible cultivars were different from the most resistant cultivar and the 155 most resistant cultivars were different from the most susceptible cultivar. However, there were 157 cultivars that were not significantly different from either extreme. The slight but significant difference between unweighted and weighted data may arise from the short time interval between $\tau_{2}$ and $\tau_{3}$. Thus, cultivars cannot be distinguished by weighted scores, because the last scoring time, likely resulting in the greatest differences between visual scorings of cultivars, had the smallest effect on AUDPC and, consequently, differences arising from the latest scores were suppressed. Despite differences between statistical properties of unweighted and weighted data, the ranking based on them is pretty similar: Spearman's correlation between mean ranks of unweighted and weighted scores of cultivars is high $\left(r_{s}=0.97, P<2.2 \times 10^{-16}\right)$. Also, Spearman's correlations between AUDPC, mean rank of unweighted scores, and arithmetic mean of unweighted scores are high (AUDPC versus mean ranks: $r_{s}=0.97$, AUDPC versus means: $r_{s}=0.97$, and mean ranks versus means: $r_{s}=0.998 ; P<2.2 \times 10^{-16}$ for each).

Correlation between replicates. Correlations between the biological replicates ranged from 0.23 to 0.66 for AIA measurements (Fig. A3). The highest correlation between the two replicates

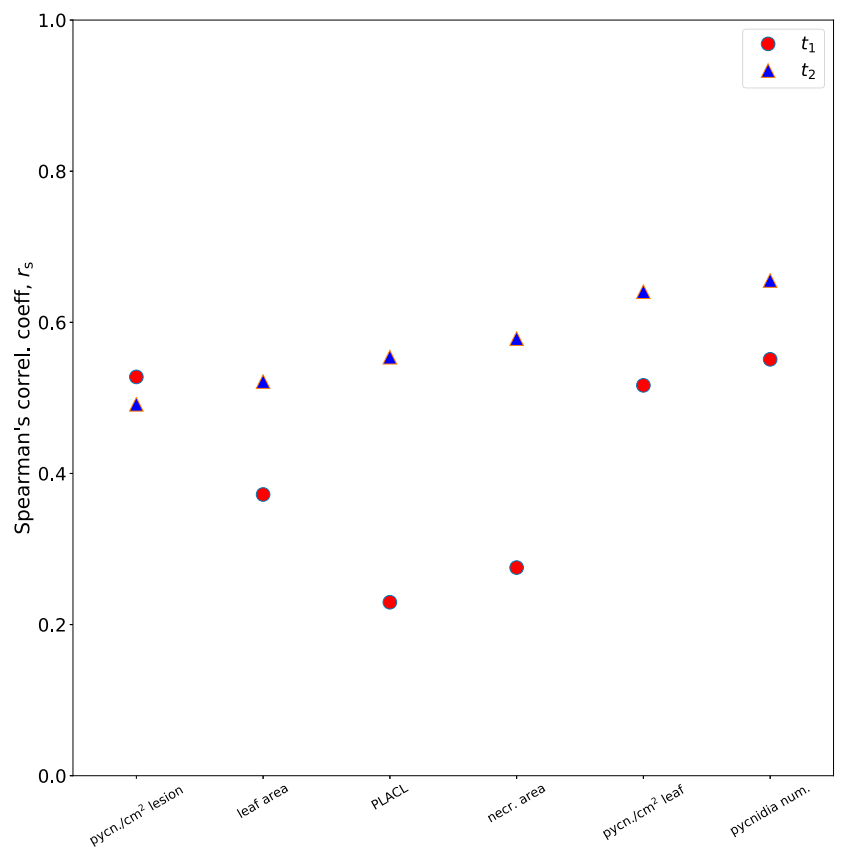

Fig. A3. Spearman's correlation coefficient between the mean values (taken over leaves belonging to the same cultivar) in two replicates for six different automated image analysis measurements. All results are highly significant $\left(P<10^{-7}\right)$. 
was found in pycnidia numbers at $t_{2}$. The lowest correlation was in PLACL at $t_{1}$. PLACL showed the largest difference in correlation coefficients between replicates. The exceptionally low correlation between replicates at $t_{1}$ for PLACL may have arisen from making the collection at a critical point in the epidemic; if the last infection cycle had been just entering the necrotic phase (as suggested in Fig. A2), there could have been a large variation between replicates by chance due to the highly variable length of the latent period for STB infection. Correlation between visual scores was not significant in first assessment but was significant and in similar range as for AIA in the second and third assessment $\left(r_{\mathrm{s}}=0.37, P=6 \times 10^{-12}\right.$ and $r_{\mathrm{s}}=$ $0.18, P=9 \times 10^{-4}$, respectively).

Spearman's correlation describes the linear relationship between rankings based on the two replicates. Moderate but highly significant correlations between replicates for each measure imply that resistance rankings based on these measures may differ considerably between replicates. We expect this to result from the shape of the quantitative resistance distributions (Figs. 2 and 3). For all three main measures of resistance (PLACL, $\rho_{\text {lesion }}$, and $\rho_{\text {leaf }}$ ), means of the measure are quite similar for all cultivars, except for a small number of cultivars at the phenotype extremes. Thus, even a small variation in these measures between replicates may result in a large change in ranking of a cultivar for one replicate to other. This is also implied by the small statistical differences between cultivars in the middle of the distributions (Figs. 2 and 3). The same arguments hold for low (and not significant) correlations between visual scores of the replicates.

Use of means versus medians in the analysis of STB resistance. Because our data were clearly nonnormal, the central tendency of the data may be better described by medians than means. Comparing medians is also biologically reasonable: total yield is more likely determined by the large number of moderately damaged wheat plants than by the few heavily diseased or dead plants. We also found stronger correlations between AUDPC and the medians of PLACL and $\rho_{\text {lesion }}$ than between AUDPC and the means of PLACL and $\rho_{\text {lesion. Nevertheless, when comparing }}$ AUDPC and $\rho_{\text {leaf }}$, we found stronger correlation between AUDPC and the means of $\rho_{\text {leaf }}$ than the medians. This may indicate that mean values better describe the way breeders traditionally assess diseases caused by necrotrophs. If this insight proves to be true, it may help us to understand and counteract the subjective nature of visual assessment with its tendency to overweight fully diseased plants and, thereby, overestimate the overall disease severity.

\section{ACKNOWLEDGMENTS}

We thank D. Dos Santos Pereira, S. Fouché, and L. Meile for help in collecting and processing leaf samples; E. Stewart for advice and guidance in using the leaf image analysis software; and $\mathrm{H}$. Zellweger for managing the wheat trial.

\section{LITERATURE CITED}

Benjamini, Y., and Hochberg, Y. 1995. Controlling the false discovery rate: A practical and powerful approach to multiple testing. J. R. Stat. Soc. B 57: 289-300.

Brading, P. A., Verstappen, E. C., Kema, G. H., and Brown, J. K. 2002. A gene-for-gene relationship between wheat and Mycosphaerella graminicola, the Septoria tritici blotch pathogen. Phytopathology 92:439-445.

Brokenshire, T. 1976. The reaction of wheat genotypes to Septoria tritici. Ann. Appl. Biol. 82:415-423.

Brown, J., Kema, G., Forrer, H.-R., Verstappen, E., Arraiano, L., Brading, P., Foster, E., Fried, P., and Jenny, E. 2001. Resistance of wheat cultivars and breeding lines to Septoria tritici blotch caused by isolates of Mycosphaerella graminicola in field trials. Plant Pathol. 50:325-338.

Brunner, P., Stefanato, F., and McDonald, B. 2008. Evolution of the CYP51 gene in Mycosphaerella graminicola: Evidence for intragenic recombination and selective replacement. Mol. Plant Pathol. 9:305-316.

Chartrain, L., Brading, P., Makepeace, J., and Brown, J. 2004. Sources of resistance to Septoria tritici blotch and implications for wheat breeding. Plant Pathol. 53:454-460.
Cools, H. J., and Fraaije, B. 2013. Update on mechanisms of azole resistance in Mycosphaerella graminicola and implications for future control. Pest Manage. Sci. 69:150-155.

Cowger, C., Hoffer, M. E., and Mundt, C. C. 2000. Specific adaptation by Mycosphaerella graminicola to a resistant wheat cultivar. Plant Pathol. 49: 445-451.

Dean, R., Van Kan, J. A. L., Pretorius, Z. A., Hammond-Kosack, K. I. M. E., Pietro, A. D. I., Spanu, P. D., Rudd, J. J., Dickman, M., Kahmann, R., Ellis, J., and Foster, G. D. 2012. The Top 10 fungal pathogens in molecular plant pathology. Mol. Plant Pathol. 13:414-430.

Deery, D., Jimenez-Berni, J., Jones, H., Sirault, X., and Furbank, R. 2014. Proximal remote sensing buggies and potential applications for field-based phenotyping. Agronomy (Basel) 4:349-379.

de Mendiburu, F. 2016. agricolae: Statistical Procedures for Agricultural Research. Online publication. https://cran.r-project.org/web/packages/agricolae/ index.html

Duncan, K. E., and Howard, R. J. 2000. Cytological analysis of wheat infection by the leaf blotch pathogen Mycosphaerella graminicola. Mycol. Res. 104:1074-1082.

Estep, L., Torriani, S., Zala, M., Anderson, N., Flowers, M., McDonald, B. A., Mundt, C., and Brunner, P. C. 2015. Emergence and early evolution of fungicide resistance in North American populations of Zymoseptoria tritici. Plant Pathol. 64:961-971.

Eyal, Z. 1992. The response of field-inoculated wheat cultivars to mixtures of Septoria tritici isolates. Euphytica 61:25-35.

Fones, H., and Gurr, S. 2015. The impact of Septoria tritici blotch disease on wheat: An EU perspective. Fungal Genet. Biol. 79:3-7.

Fraaije, B., Cools, H. J., Fountaine, J., Lovell, D. J., Motteram, J., West, J. S., and Lucas, J. 2005. Role of ascospores in further spread of QoI-resistant cytochrome b alleles (G143A) in field populations of Mycosphaerella graminicola. Phytopathology 95:933-941.

Friesen, T. L., Faris, J. D., Solomon, P. S., and Oliver, R. P. 2008. Host-specific toxins: Effectors of necrotrophic pathogenicity. Cell. Microbiol. 10:1421-1428.

Griffin, M. J., and Fisher, N. 1985. Laboratory studies on benzimidazole resistance in Septoria tritici. EPPO Bull. 15:505-511.

International Wheat Genome Sequencing Consortium. 2014. A chromosomebased draft sequence of the hexaploid bread wheat (Triticum aestivum). Science 345:1251788.

Jorgensen, L. N., Hovmoller, M. S., Hansen, J., Lassen, P., Clark, B., Bayles, R., Rodemann, B., Flath, K., Jahn, M., Goral, T., Jerzy Czembor, J., Cheyron, P., Maumene, C., De Pope, C., Ban, R., Nielsen, G. C., and Berg, G. 2014. IPM strategies and their dilemmas including an introduction to www.eurowheat.org. J. Integr. Agric. 13:265-281.

Kema, G. H., Yu, D., Rijkenberg, F. H., Shaw, M. W., and Baayen, R. P. 1996. Histology of the pathogenesis of Mycosphaerella graminicola in wheat. Phytopathology 86:777-786.

Kirchgessner, N., Liebisch, F., Yu, K., Pfeifer, J., Friedli, M., Hund, A., and Walter, A. 2017. The ETH field phenotyping platform FIP: A cablesuspended multi-sensor system. Funct. Plant Biol. 44:154-168.

Kollers, S., Rodemann, B., Ling, J., Korzun, V., Ebmeyer, E., Argillier, O., Hinze, M., Plieske, J., Kulosa, D., Ganal, M. W., and Röder, M. S. 2013a. Whole genome association mapping of Fusarium head blight resistance in European winter wheat (Triticum aestivum L.). PLoS One 8:e57500.

Kollers, S., Rodemann, B., Ling, J., Korzun, V., Ebmeyer, E., Argillier, O., Hinze, M., Plieske, J., Kulosa, D., Ganal, M. W., and Röder, M. S. 2013b. Genetic architecture of resistance to Septoria tritici blotch (Mycosphaerella graminicola) in European winter wheat. Mol. Breed. 32:411-423.

Kou, Y., and Wang, S. 2010. Broad-spectrum and durability: Understanding of quantitative disease resistance. Curr. Opin. Plant Biol. 13:181-185.

Krattinger, S. G., Lagudah, E. S., Spielmeyer, W., Singh, R. P., Huerta-Espino, J., McFadden, H., Bossolini, E., Selter, L. L., and Keller, B. 2009. A putative ABC transporter confers durable resistance to multiple fungal pathogens in wheat. Science 323:1360-1363.

Linde, C., Zhan, J., and McDonald, B. A. 2002. Population structure of Mycosphaerella graminicola: From lesions to continents. Phytopathology 92:946-955.

Madden, L., Hughes, G., and van den Bosch, F. 2007. The Study of Plant Disease Epidemics. American Phytopathological Society Press, St. Paul, MN.

Mahlein, A.-K. 2016. Plant disease detection by imaging sensors-Parallels and specific demands for precision agriculture and plant phenotyping. Plant Dis. 100:241-251.

McDonald, B. A., and Linde, C. 2002. Pathogen population genetics, evolutionary potential, and durable resistance. Annu. Rev. Phytopathol. 40: 349-379.

McDonald, B. A., and Mundt, C. C. 2016. How knowledge of pathogen population biology informs management of Septoria tritici blotch. Phytopathology 106:948-955.

Michel, V. 2001. La sélection de variétés de blé et de triticale résistantes aux maladies. Rev. Suisse Agric. 33:133-140. 
Miedaner, T., Zhao, Y., Gowda, M., Longin, C. F. H., Korzun, V., Ebmeyer, E., Kazman, E., and Reif, J. C. 2013. Genetic architecture of resistance to Septoria tritici blotch in European wheat. BMC Genomics 14:858.

Mundt, C. C. 2014. Durable resistance: A key to sustainable management of pathogens and pests. Infect. Genet. Evol. 27:446-455.

Mutka, A. M., and Bart, R. S. 2015. Image-based phenotyping of plant disease symptoms. Front. Plant Sci. 5:734.

Oliver, R. P., Friesen, T. L., Faris, J. D., and Solomon, P. S. 2012. Stagonospora nodorum: From pathology to genomics and host resistance. Annu. Rev. Phytopathol. 50:23-43.

Parlevliet, J. E. 1979. Components of resistance that reduce the rate of epidemic development. Annu. Rev. Phytopathol. 17:203-222.

Poland, J., Balint-Kurti, P. J., Wisser, R. J., Pratt, R. C., and Nelson, R. J. 2009. Shades of gray: The world of quantitative disease resistance. Trends Plant Sci. 14:21-29.

R Core Team. 2016. R: A Language and Environment for Statistical Computing. Online publication. R Foundation for Statistical Computing, Vienna. https://www.R-project.org/

Rosielle, A. 1972. Sources of resistance in wheat to speckled leaf blotch caused by Septoria tritici. Euphytica 21:152-161.

Sánchez-Vallet, A., McDonald, M. C., Solomon, P. S., and McDonald, B. A. 2015. Is Zymoseptoria tritici a hemibiotroph? Fungal Genet. Biol. 79: $29-32$.

Schindelin, J., Rueden, C. T., Hiner, M. C., and Eliceiri, K. W. 2015. The ImageJ ecosystem: An open platform for biomedical image analysis. Mol. Reprod. Dev. 82:518-529.

Shaner, G., and Finney, R. E. 1982. Resistance in soft red winter wheat to Mycosphaerella graminicola. Phytopathology 72:154-158.

Shaner, G., Finney, R. E., and Patterson, F. L. 1975. Expression and effectiveness of resistance in wheat to Septoria leaf blotch. Phytopathology 65: 761-766.

Shaw, M. 1990. Effects of temperature, leaf wetness and cultivar on the latent period of Mycosphaerella graminicola on winter wheat. Plant Pathol. 39: 255-268.

Simko, I., Jimenez-Berni, J. A., and Sirault, X. R. 2017. Phenomic approaches and tools for phytopathologists. Phytopathology 107:6-17.

Simon, M. R., and Cordo, C. A. 1998. Diallel analysis of four resistance components to Septoria tritici in six crosses of wheat (Triticum aestivum). Plant Breed. 117:123-126.

Sokal, R. R., and Rohlf, F. J. 2012. Biometry. W. H. Freeman, New York.
St.Clair, D. A. 2010. Quantitative disease resistance and quantitative resistance loci in breeding. Annu. Rev. Phytopathol. 48:247-268.

Stewart, E. L., Hagerty, C. H., Mikaberidze, A., Mundt, C. C., Zhong, Z., and McDonald, B. A. 2016. An improved method for measuring quantitative resistance to the wheat pathogen Zymoseptoria tritici using high-throughput automated image analysis. Phytopathology 106:782-788.

Stewart, E. L., and McDonald, B. A. 2014. Measuring quantitative virulence in the wheat pathogen Zymoseptoria tritici using high-throughput automated image analysis. Phytopathology 104:985-992.

Torriani, S. F., Brunner, P. C., McDonald, B. A., and Sierotzki, H. 2009. QoI resistance emerged independently at least 4 times in European populations of Mycosphaerella graminicola. Pest Manage. Sci. 65:155-162.

Torriani, S. F., Melichar, J. P., Mills, C., Pain, N., Sierotzki, H., and Courbot, M. 2015. Zymoseptoria tritici: A major threat to wheat production, integrated approaches to control. Fungal Genet. Biol. 79:8-12.

Willocquet, L., Savary, S., and Yuen, J. 2017. Multiscale phenotyping and decision strategies in breeding for resistance. Trends Plant Sci. 22:420-432.

Zadoks, J. C., Chang, T. T., and Konzak, C. F. 1974. A decimal code for the growth stages of cereals. Weed Res. 14:415-421.

Zhan, J., Mundt, C. C., Hoffer, M. E., and McDonald, B. A. 2002. Local adaptation and effect of host genotype on the rate of pathogen evolution: An experimental test in a plant pathosystem. J. Evol. Biol. 15:634-647.

Zhan, J., Mundt, C. C., and McDonald, B. A. 1998. Measuring immigration and sexual reproduction in field populations of Mycosphaerella graminicola. Phytopathology 88:1330-1337.

Zhan, J., Mundt, C. C., and McDonald, B. A. 2000. Estimation of rates of recombination and migration in populations of plant pathogens-A reply. Phytopathology 90:324-326.

Zhan, J., Pettway, R., and McDonald, B. A. 2003. The global genetic structure of the wheat pathogen Mycosphaerella graminicola is characterized by high nuclear diversity, low mitochondrial diversity, regular recombination, and gene flow. Fungal Genet. Biol. 38:286-297.

Zhan, J., Stefanato, F., and McDonald, B. A. 2006. Selection for increased cyproconazole tolerance in Mycosphaerella graminicola through local adaptation and in response to host. Mol. Plant Pathol. 7:259-268.

Zhong, Z., Marcel, T. C., Hartmann, F. E., Ma, X., Plissonneau, C., Zala, M., Ducasse, A., Confais, J., Compain, J., Lapalu, N., Amselem, J., McDonald, B. A., Croll, D., and Palma-Guerrero, J. 2017. A small secreted protein in Zymoseptoria tritici is responsible for avirulence on wheat cultivars carrying the Stb6 resistance gene. New Phytol. 214:619-631. 